\title{
Metabasites petrology and P-T evolution in the Lesser Himalaya, central Nepal
}

\author{
*L. P. Paudel ${ }^{1}$, T. Imayama ${ }^{2}$, and K. Arita $^{3}$ \\ ${ }^{1}$ Central Department of Geology, Tribhuvan University, Kirtipur, Kathmandu, Nepal \\ ${ }^{2}$ Hokkaido University Museum, Kita 10, Nishi 8, Sapporo 060-0810, Japan \\ ${ }^{3}$ Center for Chronological Research, Nagoya University, Nagoya, 464-8602, Japan \\ (*Email:lalupaudel67@yahoo.com)
}

\begin{abstract}
Petrological study was carried out for the first time on the metabasites of the Lesser Himalaya in central Nepal. The metabasites are mostly tholeiitic basalts emplaced in the clastic sediments as supracrustal dikes and sills, and later metamorphosed together with the host rocks. They contain almost a constant mineral assemblage of Ca-amphiboles + plagioclase + biotite + quartz \pm epidote \pm chlorite + (Fe-Ti oxides). Amphiboles in the form of porphyroblast show chemical zonation with actinolite/magnesiohornblende cores, tschermakite/ferro-tschermakite rims, and magnesio-hornblende margins. The cores of porphyroblasts are pre-kinematic and were probably formed prior to the Tertiary Himalayan orogeny. The porphyroblast rims and the matrix amphiboles are syn-kinematic and were formed during the Upper Main Central Thrust activity in the Tertiary period. The compositions of both the porphyroblast rims and matrix amphiboles change from actinolite in the chlorite zone to magnesiumhornblende in the biotite zone and to tschermakite/ferro-tschermakite in the garnet zone. The systematic changes in amphibole compositions as well as petrographic characteristics of metabasites confirm the classical concept of increasing metamorphic grade structurally upwards to the Upper Main Central Thrust in the Lesser Himalaya. Application of hornblende-plagioclase thermobarometry shows a coherent prograde P-T path in zoned amphiboles. The cores of amphibole porphyroblasts were formed at average peak temperature of $\sim 540^{\circ} \mathrm{C}$ and at pressure of $\sim 3 \mathrm{kbar}$. The porphyroblast rims and matrix amphiboles were recrystallized at average peak temperatures of $\sim 570^{\circ} \mathrm{C}$ in the biotite zone and $\sim 630^{\circ} \mathrm{C}$ in the garnet zone at pressure of $\sim 6 \mathrm{kbar}$. The metabasites petrology is in favor of the tectono-metamorphic models that relate the inverted metamorphism with thrusting along the Upper Main Central Thrust and coeval inversion of isotherms. It is suggested that published amphibole cooling ages from the Nepalese Lesser Himalaya based on simples, homogeneous mineralogy should be reinterpreted in view of the presence of polygenetic amphiboles with heterogeneous composition.
\end{abstract}

Keywords: Metabasites, amphiboles, thermobarometry, inverted metamorphism, Lesser Himalaya

Received: 23 December 2010

\section{INTRODUCTION}

The metamorphic core of the Himalaya in central Nepal is characterized by a sequence of inverted metamorphic zones in which the lower-grade metamorphic rocks are successively overlain by higher-grade metamorphic rocks (Le Fort 1975; Arita 1983; Pêcher and Le Fort 1986). From the Upper Main Central Thrust (Upper MCT: equivalent to MCT of Le Fort 1975) to the top of the Higher Himalaya, the kyanite zone is followed structurally upwards by the sillimanite zone. In the Lesser Himalaya, the footwall of the Upper MCT, the chlorite zone in the south (structurally lower level) is followed successively upwards (northwards) by the biotite and then garnet zones. Although metamorphic zones seem to be continuous along the N-S cross-section, a clear tectono-metamorphic discontinuity occurs along the Upper MCT (Paudel and Arita 2002). In the Higher Himalaya, the inverted metamorphism is diachronous (Pêcher and Le Fort 1986; Pêcher 1989), i.e., a kyanitegrade Barrovian-type metamorphism is overprinted by a revision accepted: 25 April 2011

later lower-P/higher- $\mathrm{T}$ sillimanite-grade metamorphism. The kyanite-grade regional metamorphism may be either an Eocene event (Eohimalayan stage) resulted from the crustal thickening after India-Asia collision (Le Fort 1975; Pêcher 1989) or may be an early Paleozoic event (Gehrels et al. 2006). The sillimanite-grade metamorphism in the Higher Himalaya is related to the decompression during exhumation (Pognante and Benna 1993) and leucogranite intrusion associated with the Upper MCT activity in the Neogene (Neohimalayan stage).

Contrary to that in the Higher Himalaya, the metamorphic evolution in the Lesser Himalaya is not always clear because of its lower-grade condition, lack of appropriate mineral assemblages, and relatively finegrained nature of the rocks. Some of the issues such as polymetamorphism, pre-collisional and post-collisional thermal events, and the nature and origin of the inverted metamorphism are yet to be clarified. The inverted metamorphism, in some sections, seems to be a primary 


\section{P. Paudel et al.}

pattern acquired during the Upper MCT movement (Le Fort 1975; Arita 1983; Guillot and Allemand 2002; Paudel and Arita 2006a) while, in other sections, it is a secondary pattern formed by post-metamorphic deformation (Searle and Rex 1989; Hubbard 1996; Harrison et al. 1998; Catlos et al. 2001; Bollinger et al. 2004). Some authors also argue that the Lesser Himalaya suffered a polymetamorphic evolution (Johnson and Oliver 1990; Paudel and Arita 2000; 2006a). In this context, it is important to look for complementary evidence to have clearer understanding of the metamorphism in the Lesser Himalaya and to prefer any of above models for the Himalayan metamorphic evolution.

A commonly found lithology in the northern part of the Lesser Himalaya of central Nepal is layers of metabasites intercalated within metasediments. Unlike extensive studies on metapelites, no studies have been made on those metabasites. Metabasites are mineralogically more sensitive to variation in pressure and temperature and may record metamorphic information which is not found in equivalent metasediments (Miyashiro 1973). Phase relationships in metabasites are dependent on metamorphic grade (Graham 1974; Rasse 1974; Brown 1977; Holland and Richardson 1979; Liard and Albee 1981), and amphiboles in metabasites have high potential for pressuretemperature path reconstruction (Blundy and Holland 1990; Triboulet et al. 1992; Zenk and Schulz 2004). The present work is aimed at studying the metabasites from the Lesser Himalaya in the Modi Khola section, central Nepal to provide complementary insights into the nature and history of metamorphism in the Lesser Himalaya.

\section{GEOLOGICAL SETTINGAND OCCURRENCE - OF METABASITES}

Central Nepal can be divided into four tectonic zones; from south to north, the Sub-Himalaya, the Lesser Himalaya $(\mathrm{LH})$, the Higher Himalaya $(\mathrm{HH})$ and the Tibetan-Tethys Himalaya (Gansser 1964) (Fig. 1) . The LH is a fold-and-

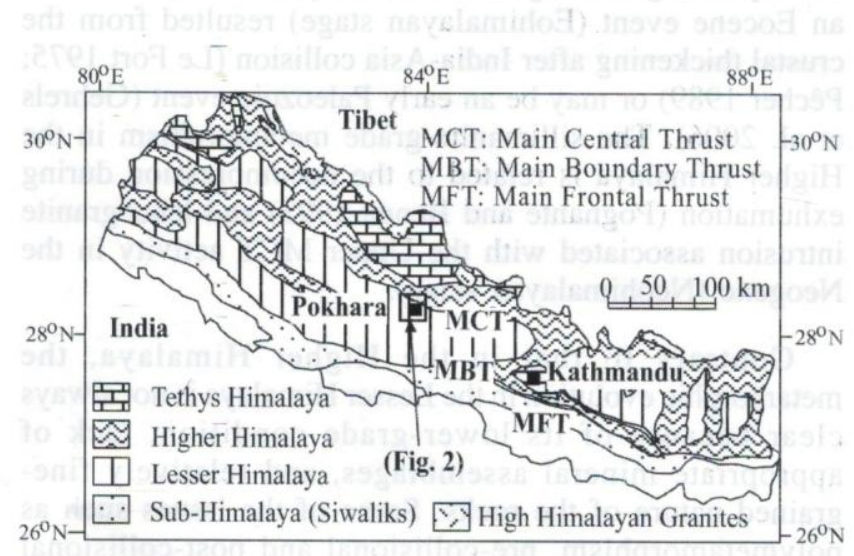

Fig. 1: Generalized tectonic map of the Nepal Himalaya showing the location of the present study area (modified after Upreti 1999) thrust belt bounded by the Main Boundary Thrust (MBT) in the south and by the Upper MCT in the north. The detailed tectono-stratigraphy of the LH in the study area is found elsewhere (Sakai 1985; Dhital et al. 1998; Paudel and Arita 2000). Here, only the brief geological outline of the LH north of the Bari Gad-Kali Gandaki Fault, referred as the inner LH (Arita et al. 1982) is described. The inner LH comprises low- to medium-grade metasedimentary rocks of the Nawakot Complex ranging in age from the late Precambrian to early Paleozoic (Stöcklin 1980) and is further divided into three tectonic zones from south to north, viz., the Thrust Sheet I (TS I), Thrust Sheet II (TS II), and the MCT zone (Fig. 2). The Phalebas Thrust (PT) separates TS I from II, and the Lower MCT separates the TS II from the MCT zone. The LH is overthrust by the HH along the Upper MCT.

The TS I comprises the middle part of Nawakot Complex (Stöcklin 1980) viz. the Nourpul Formation (phyllite and quartzite), Dhading Dolomite (dolomite and slate), and the Benighat Slate (slate and limestone). The TS II consists of the Kunchha Formation (phyllite, metasandstone), Fagfog Quartzite (quartzite, phyllite) and Dandagaon Phyllite (phyllite, metasandstone) belonging to the lower part of the Nawakot Complex. The MCT zone comprises intercalation of garnetiferous pelitic, psammitic and carbonaceous schists, and mylonitic augen gneisses. The MCT zone here includes only the footwall rocks of the Upper MCT whose protoliths were of the LH affinity (Imayama and Arita 2007), and should not be confused with others' definition from structural point of view that includes also a part of the hanging wall (the lower part of the $\mathrm{HH}$ ) into the MCT zone.

Sheet-like bodies of metabasites are found intercalated within the metasediments of the lower part of the Nawakot Complex. These metabasites are abundant in the TS II and the MCT zone (Fig. 2). In some places they range in thickness from a few meters to tens of meters and extend hundreds of meters along the strike. But in other places, they are thin (less than a meter) and pinch-out within a few meters. They are green to dark green, very hard and massive, medium- to coarse-grained, often foliated and deformed along with the associated metasediments. Foliation is strongly developed in relatively thin and finegrained bodies compared to that in relatively thick and coarse-grained bodies. The contact with the country rocks is often sharp and concordant. Similar rocks in the Indian $\mathrm{LH}$ are dated at $1907 \pm 91 \mathrm{Ma}$ based on whole rock $\mathrm{Rb}-\mathrm{Sr}$ isochron method (Ahmad et al. 1999). The metabasites may be basic lava flows or supracrustal dikes and sills emplaced into the clastic sediments in the Precambrian.

\section{DEFORMATIONAND METAMORPHISM}

Mainly two prominent foliations can be observed in the metasediments of the inner belt of the LH (Pêcher 1977; Paudel and Arita 2000). The older one is a bedding-parallel foliation $\left(\mathrm{S}_{1}=\mathrm{S}_{0}\right)$ predating the Upper MCT activity and can 
(a)

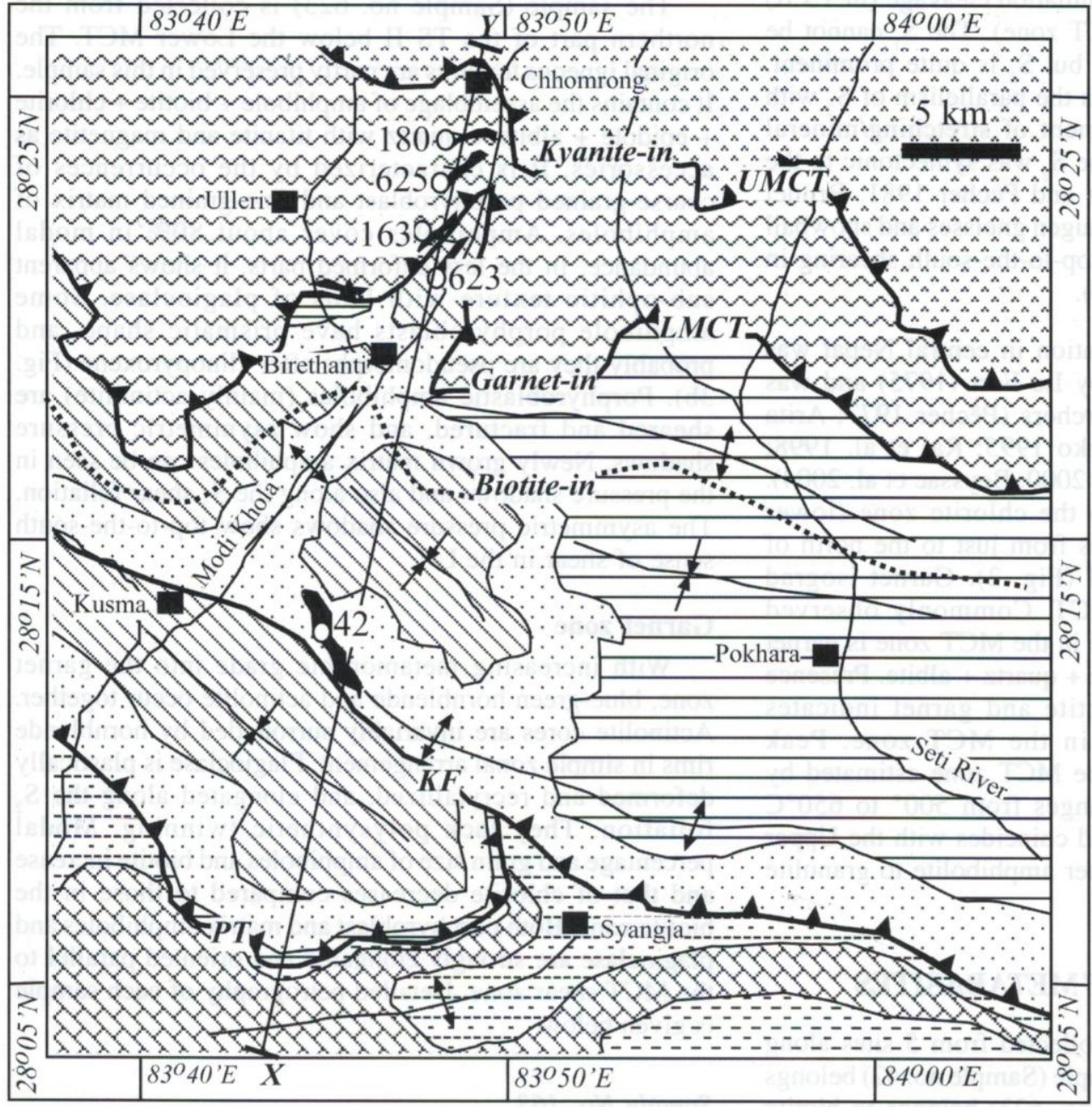

Higher Himalaya

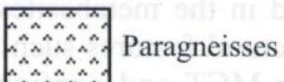

\section{Lesser Himalaya}

MCT zone

Pelitic and psammitic schits, augen gneiss, marble

TS I and TS II

:-:-- Benighat Slate

- (slate, limestone)

Dhaling Dolomite

25 (dolomite, slate)

\begin{tabular}{ll} 
Nourpul Formation \\
\hdashline-1
\end{tabular}

9andagaon Phyllite (phyllite, quartzite)

\begin{tabular}{l}
$\begin{array}{l}\text { Fagfog Quartzite } \\
\text { (quartzite, phyllite) }\end{array}$ \\
$\begin{array}{l}\text { Kunchha Formation } \\
\text { (phyllite, metasandstone) }\end{array}$ \\
\hline
\end{tabular}

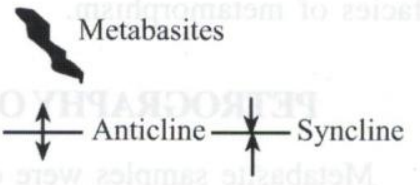

0163 Sample site and number

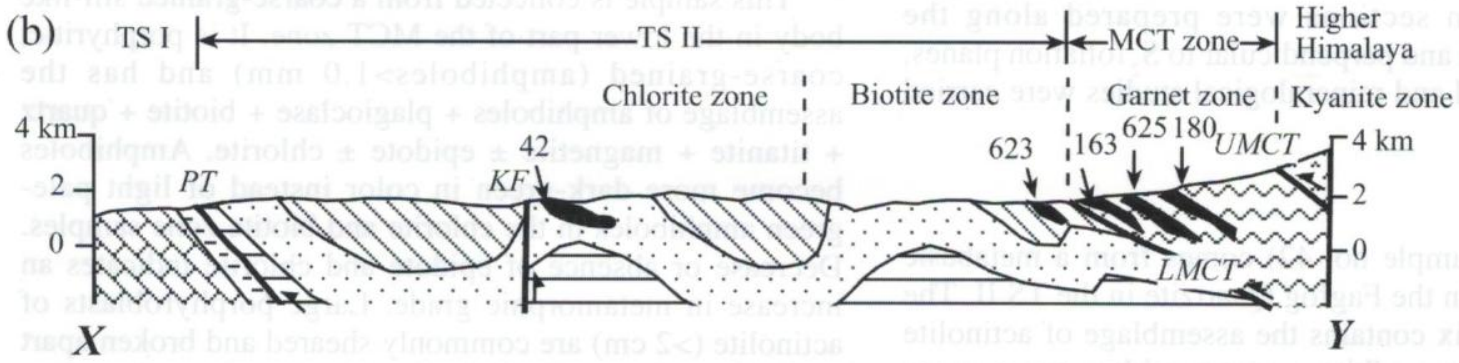

Fig. 2: (a) Geological map of the Pokhara-Kusma area (modified after Paudel and Arita 2000). UMCT: Upper Main Central Thrust, LMCT: Lower Main Central Thrust, KF: Kusma Fault, PT: Phalebas Thrust, TS I: Thrust sheet I, TS II: Thrust sheet II, MCT zone: Main Central Thrust zone. X-Y: line of cross-section. Biotite isograd is shown by dotted line. Garnet and kyanite isograds coincide with the Lower and Upper MCTs, respectively. (b) Geological cross-section along X-Y 


\section{P. Paudel et al.}

be observed in the very-low grade rocks of the TS II. The younger one $\left(\mathrm{S}_{2}\right)$ is either a crenulation cleavage (in TS II) or a shear foliation (in the MCT zone). The $S_{1}$ cannot be recognized in the metabasites, but $S_{2}$ is quite prominent. Microstructural features such as the parallelism of $\mathrm{S}_{2}$ with the Upper MCT and confinement of stretching/mineral lineations on $\mathrm{S}_{2}$ indicate that the $\mathrm{S}_{2}$ was syntectonic to the Upper MCT activity (Bouchez and Pêcher 1981; Brunel 1986). Mylonitic S-C fabric in augen gneisses and snowball garnet in metapelites indicate top-to-the-south shearing in the LH (Paudel and Arita 2000).

Inverted metamorphic zonation in central Nepal was first reported in this section by Le Fort (1975) and was endorsed by subsequent researchers (Pêcher 1977; Arita 1983; Macfarlane 1995; Kaneko 1995; Rai et al. 1998; Guillot, 1999; Paudel and Arita 2000; Beyssac et al. 2004). The southern part belongs to the chlorite zone (lower epizone). Biotite isograd passes from just to the north of Pokhara and Kusma in TS II (Fig. 2). Garnet isograd coincides with the Lower MCT. Commonly observed metapelitic mineral assemblage in the MCT zone is garnet + biotite + muscovite + chlorite + quartz + albite. Presence of S-shaped inclusion in biotite and garnet indicates syntectonic metamorphism in the MCT zone. Peak metamorphic temperature in the MCT zone estimated by garnet-biotite thermometry ranges from $500^{\circ}$ to $650^{\circ} \mathrm{C}$ (Kaneko 1995). Kyanite isograd coincides with the Upper MCT. The HH rocks show upper amphibolite to granulite facies of metamorphism.

\section{PETROGRAPHY OF METABASITES}

Metabasite samples were collected from 5 sites along the Modi Khola Valley. One sample (Sample no. 42) belongs to chlorite zone, one (Sample no. 623) belongs to biotite zone and three (Sample nos. 163, 625, and 180) belong to garnet zone. Thin sections were prepared along the stretching lineation and perpendicular to $\mathrm{S}_{2}$ foliation planes, and microstructural and mineralogical studies were carried out on them.

\section{Chlorite zone}

The sample (Sample no. 42) comes from a metabasic sill-like body within the Fagfog Quartzite in the TS II. The recrystallized matrix contains the assemblage of actinolite + chlorite + epidote + albite + quartz with some opaques (ilmenite, magnetitie) as accessories (Fig. 3a). Needleshaped actinolite and chlorite define the $\mathrm{S}_{2}$ foliation. Relic phenocrysts of plagioclase (albite) are subhedral to anhedral and range in size from 0.5 to $3 \mathrm{~mm}$. They are characterized by clear polysynthetic twinning and are frequently sericitized. Epidote and quartz occur as finegrained aggregates. Porphyroblastic amphiboles do not exist in this sample.

\section{Biotite zone}

The sample (Sample no. 623) is collected from the northern part of the TS II below the Lower MCT. The original igneous textures are partly preserved in this sample. It contains the assemblage of amphibole + biotite + chlorite + epidote + albite + quartz with titanite and magnetite as accessories. It is characterized by the occurrences of coarse-grained porphyroblast and fine-grained matrix of amphiboles. Amphiboles cover about $80 \%$ in modal abundance. In the less deformed parts, it shows apparent sub-ophitic texture with laths of plagioclase. Some amphibole porphyroblasts have prismatic shape, and probably they are pseudomorphs after clinopyroxene (Fig. 3b). Porphyroblastic amphiboles (mainly actinolite) are sheared and fractured, and show asymmetric pressure shadows. Newly grown matrix amphiboles-can be seen in the pressure shadows and also along the $\mathrm{S}_{2}$ shear foliation. The asymmetric pressure shadows show top-to-the-south sense of shear in the LH.

\section{Garnet zone}

With increasing metamorphic grade into the garnet zone, blue-green hornblende and actinolite occur together. Actinolite cores are invariably surrounded by hornblende rims in simple zonal arrangement. Plagioclase is plastically deformed and recrytallized, and elongated along the $\mathrm{S}_{2}$ foliation. They lack polysynthetic twinning. Modal percentage and grain size of amphiboles and biotite increase and that of chlorite decreases compared to those in the biotite zone. Both porphyroblast and matrix amphiboles and plagioclase are strongly elongated and oriented parallel to the MCT shear zone. Detailed petrography of each sample is given below.

\section{Sample No. 163}

This sample is collected from a coarse-grained sill-like body in the lower part of the MCT zone. It is porphyritic, coarse-grained (amphiboles $>1.0 \mathrm{~mm}$ ) and has the assemblage of amphiboles + plagioclase + biotite + quartz + titanite + magnetite \pm epidote \pm chlorite. Amphiboles become more dark-green in color instead of light palegreen amphiboles in the chlorite and biotite zone samples. Decrease or absence of epidote and chlorite indicates an increase in metamorphic grade. Large porphyroblasts of actinolite $(>2 \mathrm{~cm})$ are commonly sheared and broken apart by $\mathrm{S}_{2}$ shear planes (Fig. 3c). Hornblende commonly occurs as patches within the sheared porphyroblasts, as overgrown rims of porphyroblasts and as recrystallized grains in matrix (Figs. 3c and 3d). Late stage alteration along cracks and cleavages is common. Syn-kinematic needle-shaped amphibole grains in matrix are oriented parallel to the $S_{2}$ shear plane (Fig. 3c). Plagioclase is much more deformed and has sutured grain boundary with the quartz and amphiboles. They have no twining. 
(a) Chlorite zone

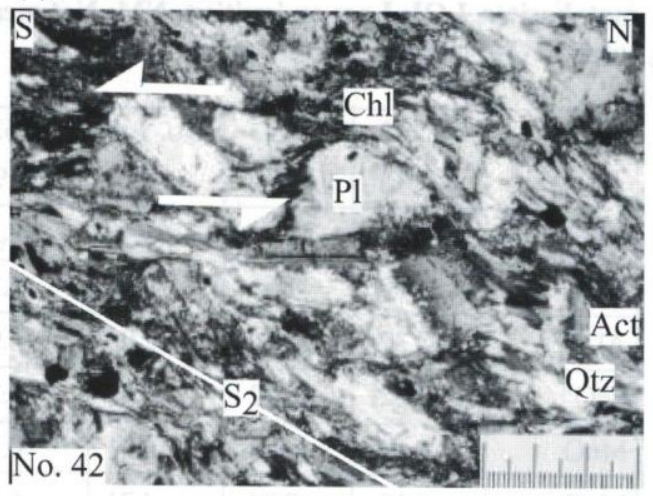

(c) Garnet zone

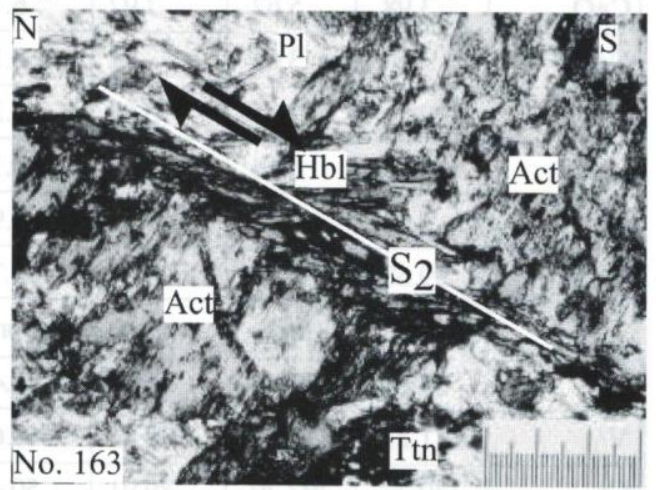

(e) Garnet zone

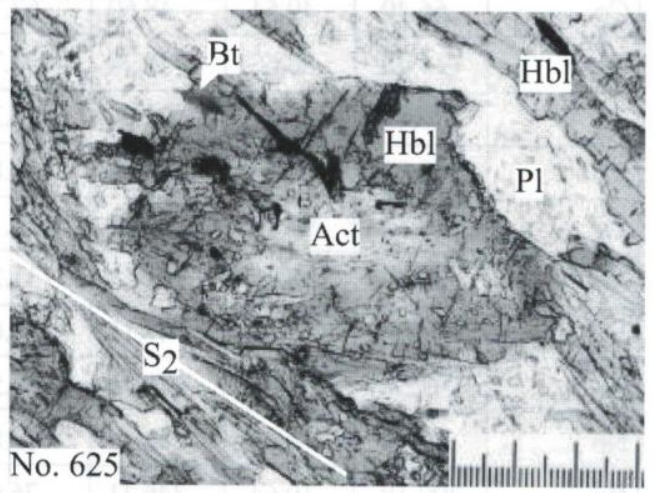

(b) Biotite zone

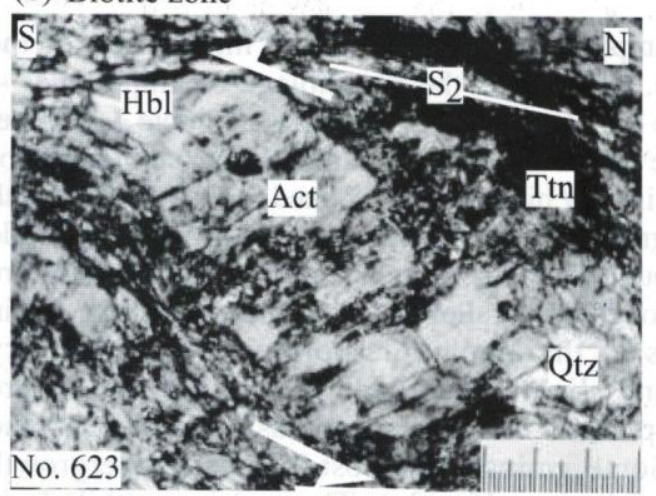

(d) Garnet zone

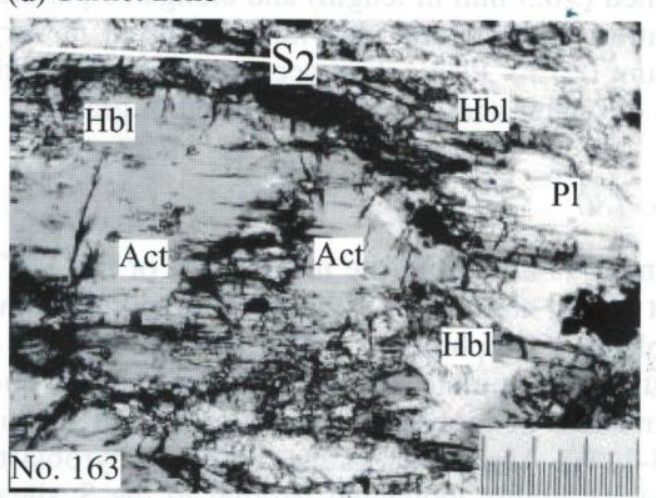

(f) Garnet zone

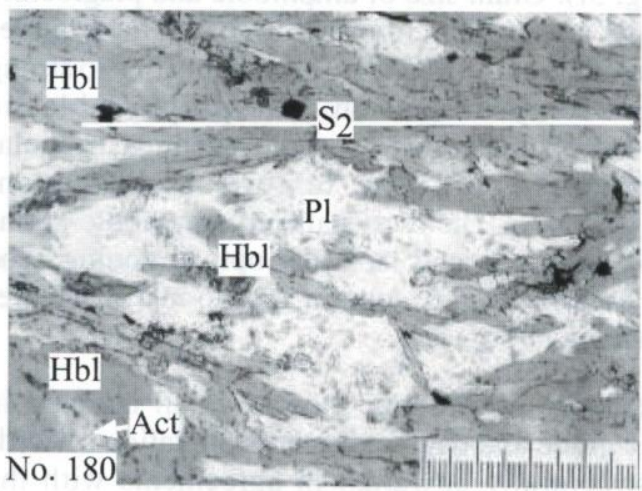

Fig. 3: Photomicrographs of metabasites. (a) Sample No. 42 from the chlorite zone is dominated by porphyroclastic (igneous relics) plagioclase, chlorite and quartz. Fine-grained recrastallized actinolite occurs only along $S_{2}$ foliation. (b) Sample No. 623 from biotite zone contains sheared actinolite porphyroblast with asymmetric pressure shadows. Dark-green hornblende is present as overgrown rim of porphyroblast in the pressure shadows and matrix along $\mathrm{S}_{2}$ foliation. Well-preserved prismatic shape of the porphyroblast indicates that the actinolite is a pseudomorph after clinopyroxene. (c) Sample No. 163 from the lower part of the garnet zone (MCT zone) contains large, highly sheared porphyroblasts of actinolite. Patchy intergrowth of blue-green hornblende (Hbl) and actinolite (Act) within amphibole porphyroblast is common. Needle-shaped synkinematic hornblende are strongly oriented parallel to the shear plane $\left(\mathrm{S}_{2}\right)$. (d) Some porphyroblasts in Sample No. 163 show acicular overgrowth of hornblende along $\mathrm{S}_{2^{*}}$ (e) Sample No. 625 from middle part of the garnet zone contains zoned porphyroblast with actinolite cores and hornblende rims. Overgrowth of rims across the foliation is observed in some porphyroblasts. Matrix is relatively coarse-grained and strongly oriented parallel to the $S_{2}$ shear foliation. (f) Sample No. 180 from the middle part of the garnet zone (MCT zone) is highly sheared compared to the other samples. Amphibole porphyroblasts are slightly zoned with patches of actinolite in the cores and hornblende in the rim (bottom left corner). Plagioclase porphyroblast show pokilitic texture with inclusions of recrystallized hornblende (centre). Act: Actinolite, Hbl: Hornblende, Chl: Chlorite, Bt: Biotite, PI: Plagioclase, Qtz: Quartz, Ttn: Titanite. $S_{2}$ : Shear foliation parallel to the Upper MCT. 1 small division of scale is $0.01 \mathrm{~mm}$ 


\section{P. Paudel et al.}

\section{Sample No. 625}

This sample is collected from the middle part of the MCT zone. It contains the assemblage of amphiboles + plagioclase + biotite + quartz + titanite \pm epidote \pm chlorite Amphibole is dark-green in color. Modal abundance of amphibole is very high $(>80 \%)$. Zoned porphyroblasts with light pale-green actinolite cores and dark-green hornblende rims are abundant in the sample (Fig. 3e). Some grains are strongly elongated parallel to the $\mathrm{S}_{2}$ shear foliation. Grain boundaries of the amphibole porphyroblasts are very irregular and slightly resorbed. The porphyroblasts are mostly of poiklitic nature with inclusions of quartz and opaques. Some grains show post-kinematic overgrowth across the foliation (Fig. 3e). The matrix amphibole is coarse-grained ( $>0.3 \mathrm{~mm}$ in length) and elongated parallel to the foliation. Epidote and chlorite are present in very minor amount $(<5 \%)$. Plagioclase occurs as inclusion-rich (inclusions of quartz and amphiboles) anhedral grains.

\section{Sample No. 180}

This sample is also from the middle part of the MCT zone, about $500 \mathrm{~m}$ north from Sample No. 625. It contains the assemblage of amphiboles + plagioclase + biotite + quartz + titanite \pm epidote. Chlorite does not occur and modal abundance of biotite is significantly high in this sample ( 5-10\%). Amphibole grains (both porphyroblasts and matrix) are highly elongated parallel to the $S_{2}$ shear foliation (Fig. 3f). Grain size of amphibole and plagioclase porphyroblasts are smaller compared to that in the samples from the lower part of the MCT zone, probably due to strong shearing and recrystallization. The porphyroblastic amphiboles are characterized by patchy zoning, irregular grain boundaries, poikilitic texture, and frequent overgrowth across $\mathrm{S}_{2}$. The recrystallized amphibole grains in the matrix are clean and lack any optical zoning. The plagioclase porphyroblasts contain inclusions of needleshaped amphiboles (Fig. 3f).

The metabasite mineralogy shows overall increase in metamorphic grade and degree of recrystallization towards the north (structurally upwards) in agreements with the northward increasing metamorphism shown by mineralogy of metapelites.

\section{WHOLE ROCK CHEMISTRY}

To have an idea about the whole rock chemistry, X-Ray fluorescence (XRF) analysis was carried out at the Hokkaido University, Japan using a PANalytical MagiXpro. The analytical procedures were followed after Tanaka and Orihashi (1997). A series of rock reference samples provided by the Geological Survey of Japan were used as a standard material to set up the calibration lines. The analytical data for 10 major elements and 20 trace elements are shown in Table 1.
Table 1: Whole (bulk) rock chemical compositions of metabasites. LOI: Loss on ignition, NM: Not measured, FeO*: total $\mathrm{FeO}+\mathrm{Fe}_{2} \mathrm{O}_{3}$ measured as $\mathrm{FeO}$.

\begin{tabular}{|c|c|c|c|c|c|}
\hline Sample & $\begin{array}{l}\text { No. } 42 \\
\text { (wt } \% \text { ) }\end{array}$ & $\begin{array}{r}\text { No. } 623 \\
\text { (wt\%) }\end{array}$ & $\begin{array}{r}\text { No. } 163 \\
\text { (wt\%) }\end{array}$ & $\begin{array}{r}\text { No. } 625 \\
\text { (wt } \% \text { ) }\end{array}$ & $\begin{array}{r}\text { No. } 180 \\
\text { (wt } \%)\end{array}$ \\
\hline $\mathrm{SiO} 2$ & 50.74 & 52.64 & 50.10 & 48.39 & 51.93 \\
\hline $\mathrm{TiO} 2$ & 1.89 & 1.52 & 2.82 & 2.23 & 2.36 \\
\hline $\mathrm{Al} 2 \mathrm{O} 3$ & 15.32 & 14.22 & 12.08 & 14.94 & 12.13 \\
\hline $\mathrm{FeO}^{*}$ & 11.62 & 10.22 & 12.95 & 14.27 & 15.32 \\
\hline $\mathrm{MnO}$ & 0.19 & 0.24 & 0.21 & 0.17 & 0.19 \\
\hline $\mathrm{MgO}$ & 8.10 & 7.04 & 4.71 & 6.02 & 3.89 \\
\hline $\mathrm{CaO}$ & 3.98 & 5.62 & 7.92 & -6.14 & 7.06 \\
\hline $\mathrm{Na} 2 \mathrm{O}$ & 3.64 & 3.65 & 3.72 & 4.19 & 3.39 \\
\hline $\mathrm{K} 2 \mathrm{O}$ & 2.51 & 2.45 & 0.68 & 0.31 & 1.24 \\
\hline $\mathrm{P} 2 \mathrm{O} 5$ & 0.18 & 0.16 & 0.58 & 0.17 & 0.25 \\
\hline LOI & 1.50 & 1.30 & 0.87 & 0.91 & 0.90 \\
\hline \multirow[t]{2}{*}{ Total } & 99.68 & 99.05 & 96.64 & 97.73 & 98.64 \\
\hline & (ppm) & (ppm) & (ppm) & (ppm) & (ppm) \\
\hline Sc & 35.69 & 35.42 & 35.94 & 28.43 & 28.53 \\
\hline V & 321.27 & 232.33 & 300.82 & 411.63 & 361.36 \\
\hline $\mathrm{Cr}$ & 330.27 & 297.70 & 89.91 & 50.47 & 52.81 \\
\hline Co & 66.40 & 49.54 & 48.60 & 67.72 & 50.17 \\
\hline $\mathrm{Ni}$ & 124.48 & 80.65 & 30.74 & 37.71 & 17.57 \\
\hline $\mathrm{Cu}$ & 186.49 & 28.61 & 23.04 & 5.30 & 16.37 \\
\hline $\mathrm{Rb}$ & 57.01 & 30.84 & 38.08 & 10.89 & 49.16 \\
\hline $\mathrm{Zn}$ & 130.13 & 90.78 & 123.37 & 120.18 & 142.43 \\
\hline $\mathrm{Sr}$ & 89.59 & 118.42 & 332.37 & 131.21 & 194.00 \\
\hline \begin{tabular}{|l}
$\mathrm{Y}$ \\
\end{tabular} & 26.00 & 21.24 & 47.17 & 30.66 & 31.01 \\
\hline $\mathrm{Zr}$ & 137.58 & 52.09 & 245.36 & 168.52 & 165.47 \\
\hline $\mathrm{Nb}$ & 12.94 & 6.78 & 40.13 & 13.87 & 12.69 \\
\hline $\mathrm{Ba}$ & 290.18 & 19.64 & 346.11 & 76.01 & 349.06 \\
\hline $\mathrm{Pb}$ & 5.99 & 21.74 & 17.21 & 13.07 & 14.70 \\
\hline Th & 6.17 & 4.26 & 6.43 & 9.27 & 8.77 \\
\hline $\mathrm{Ga}$ & 18.28 & 16.03 & 21.51 & 22.52 & 25.28 \\
\hline $\mathrm{La}$ & 0.99 & $\mathrm{NM}$ & 24.17 & 30.56 & 17.55 \\
\hline $\mathrm{Ce}$ & 48.67 & 26.63 & 64.55 & 35.01 & 41.48 \\
\hline Ti & 11331 & 9104 & 16888 & 13391 & 14127 \\
\hline $\mathrm{U}$ & $\mathrm{NM}$ & $\mathrm{NM}$ & 0.47 & $\mathrm{NM}$ & 1.19 \\
\hline $\mathrm{FeO} / \mathrm{MgO}$ & 1.44 & 1.45 & 2.75 & 2.37 & 3.94 \\
\hline $\mathrm{Zr} / \mathrm{TiO} 2$ & 0.007 & 0.003 & 0.009 & 0.008 & 0.007 \\
\hline $\mathrm{Nb} / \mathrm{Y}$ & 0.498 & 0.319 & 0.851 & 0.452 & 0.409 \\
\hline
\end{tabular}




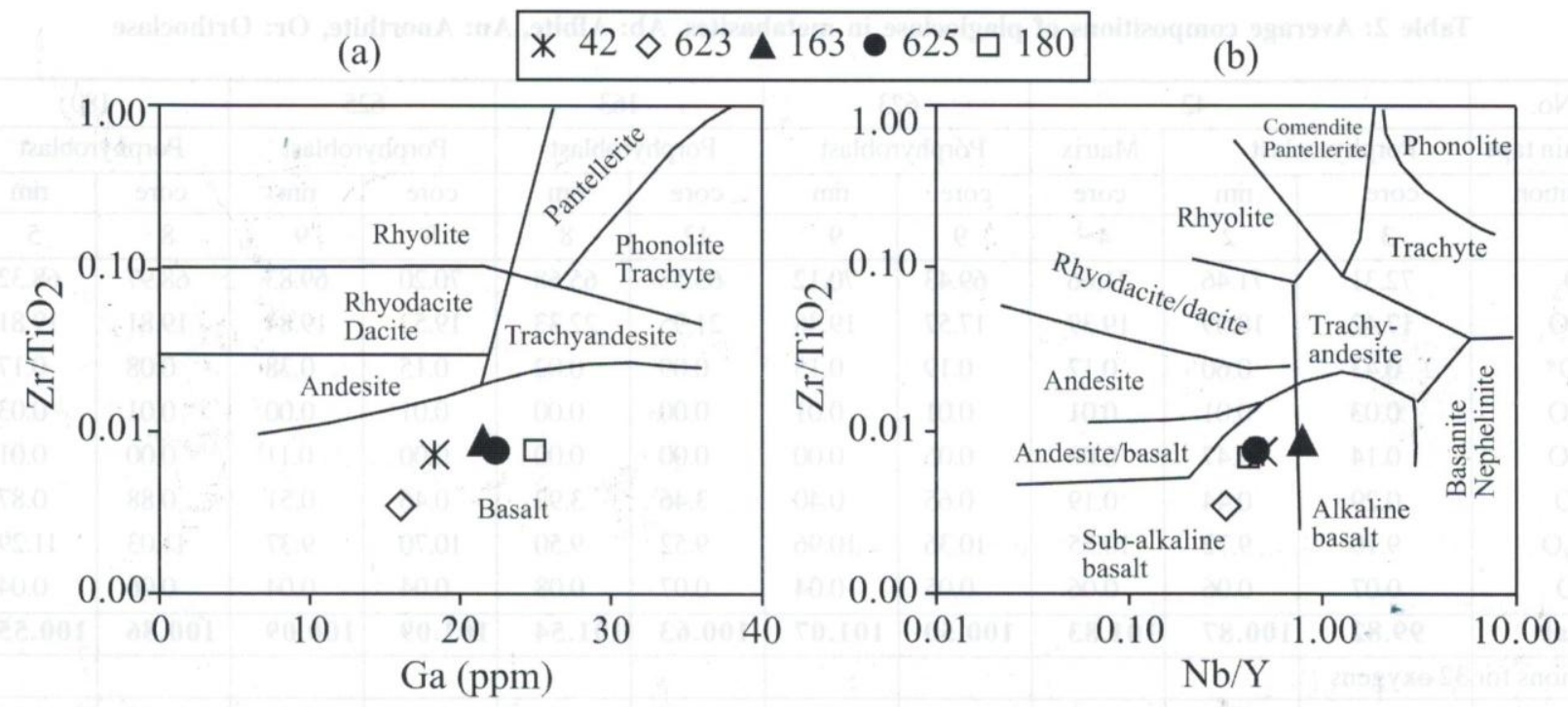

Fig. 4. Plot of the whole rock composition in the discrimination diagram of Winchester and Floyd (1977)

(a)

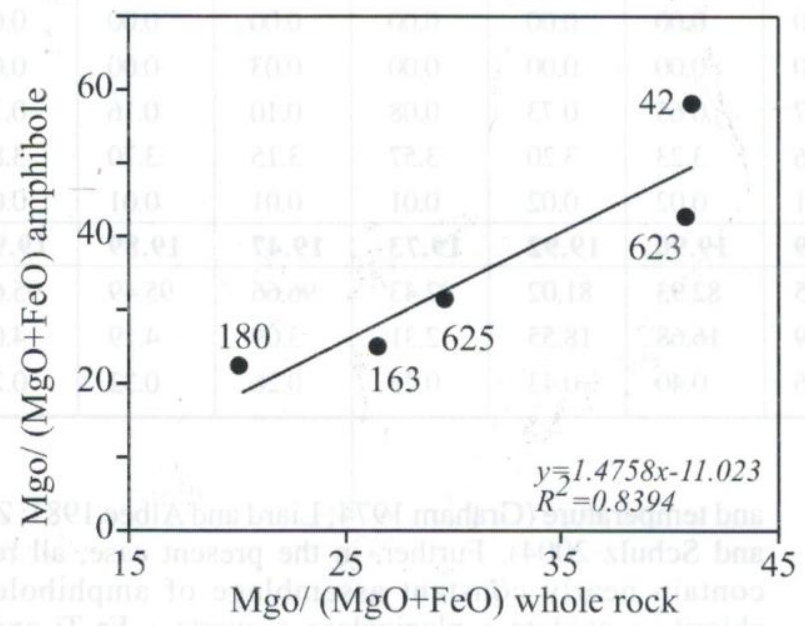

(c)

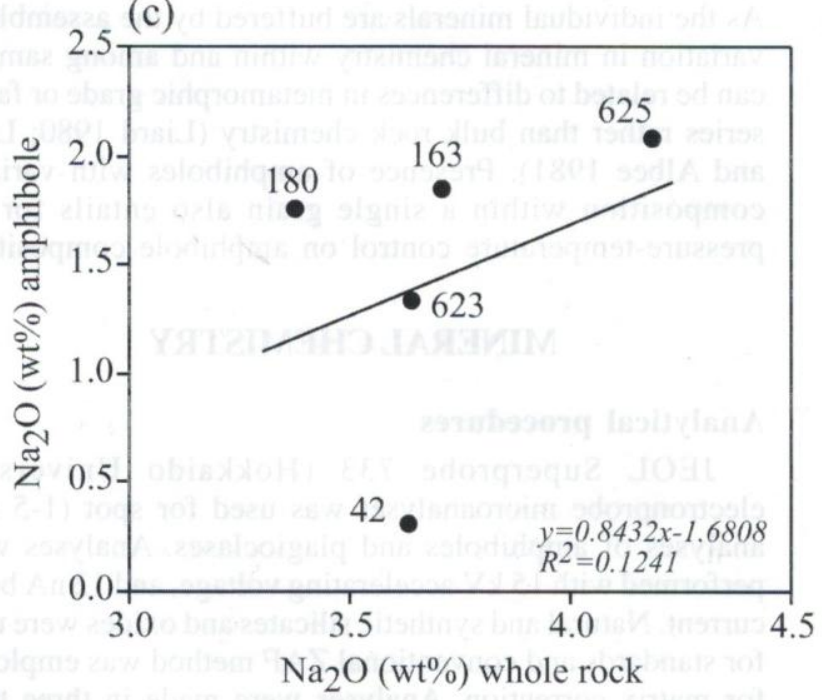

(b)

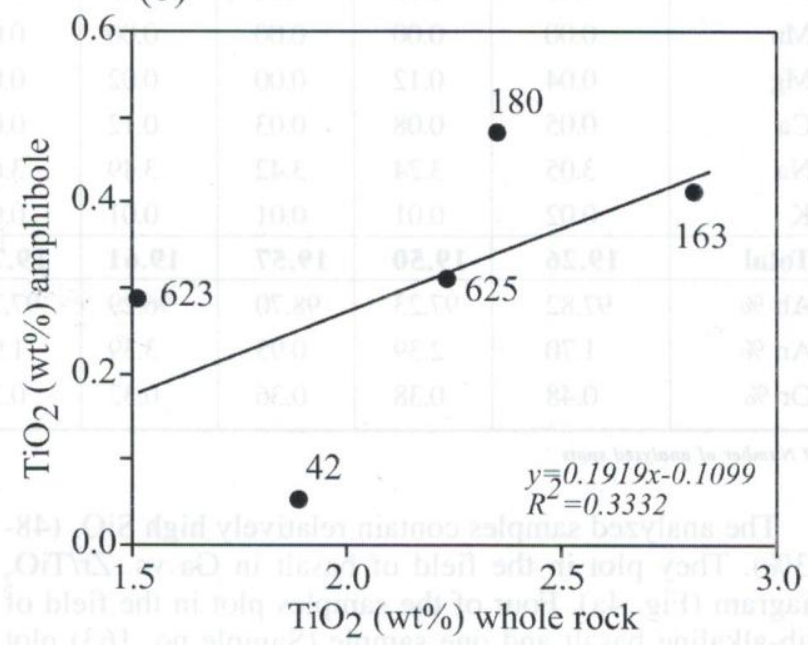

(d)

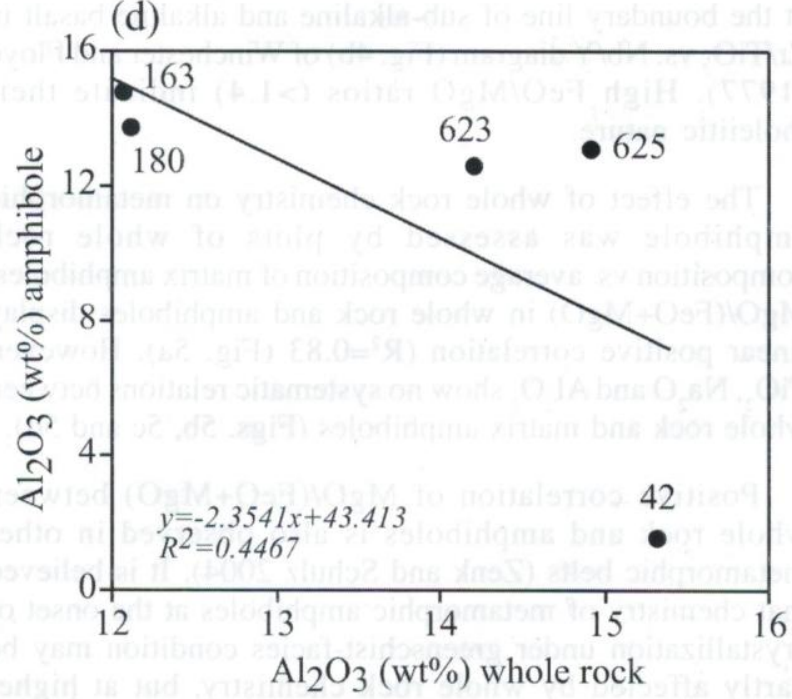

Fig. 5: Plot of whole rock composition vs. average composition of matrix amphiboles 


\section{P. Paudel et al.}

Table 2: Average compositions of plagioclase in metabasites. Ab: Albite, An: Anorthite, Or: Orthoclase

\begin{tabular}{|c|c|c|c|c|c|c|c|c|c|c|c|}
\hline \multirow{3}{*}{\begin{tabular}{|l} 
S. No. \\
Grain tape \\
Position \\
\end{tabular}} & \multicolumn{3}{|c|}{42} & \multirow{2}{*}{\multicolumn{2}{|c|}{$\begin{array}{c}623 \\
\text { Porphyroblast }\end{array}$}} & \multirow{2}{*}{\multicolumn{2}{|c|}{$\begin{array}{c}163 \\
\text { Porphyroblast }\end{array}$}} & \multirow{2}{*}{\multicolumn{2}{|c|}{$\begin{array}{c}625 \\
\text { Porphyroblast }\end{array}$}} & \multirow{2}{*}{\multicolumn{2}{|c|}{$\begin{array}{c}180 \\
\text { Porphyroblast }\end{array}$}} \\
\hline & \multicolumn{2}{|c|}{ Porphyroclast } & \multirow{2}{*}{$\begin{array}{c}\text { Matrix } \\
\text { core }\end{array}$} & & & & & & & & \\
\hline & core & rim & & core & rim & core & rim & core & rim & core & rim \\
\hline \# & 3 & 2 & 4 & 9 & 9 & 12 & 8 & 8 & 9 & 8 & 5 \\
\hline $\mathrm{SiO}_{2}$ & 72.31 & 71.46 & 71.66 & 69.43 & 70.12 & 65.53 & 65.68 & 70.20 & 69.83 & 68.97 & 68.32 \\
\hline $\mathrm{Al}_{2} \mathrm{O}_{3}$ & 17.42 & 18.09 & 19.39 & 17.57 & 19.38 & 21.95 & 22.33 & 19.52 & 19.84 & 19.81 & 19.81 \\
\hline $\mathrm{FeO}^{*}$ & -0.43 & 0.60 & 0.17 & 0.19 & 0.15 & 0.09 & 0.02 & 0.15 & 0.38 & 0.08 & 0.17 \\
\hline $\mathrm{MnO}$ & 0.03 & 0.01 & 0.01 & 0.01 & 0.01 & 0.00 & 0.00 & 0.01 & 0.00 & 0.01 & 0.03 \\
\hline $\mathrm{MgO}$ & 0.14 & 0.47 & 0.00 & 0.06 & 0.00 & 0.00 & 0.00 & 0.00 & 0.11 & 0.00 & 0.01 \\
\hline $\mathrm{CaO}$ & 0.29 & 0.44 & 0.19 & 0.65 & 0.40 & 3.46 & 3.92 & 0.46 & 0.51 & 0.88 & 0.87 \\
\hline $\mathrm{Na}_{2} \mathrm{O}$ & 9.10 & 9.72 & 10.35 & 10.36 & 10.96 & 9.52 & 9.50 & 10.70 & 9.37 & 11.03 & 11.29 \\
\hline $\mathrm{K}_{2} \mathrm{O}$ & 0.07 & 0.06 & 0.06 & 0.05 & 0.04 & 0.07 & 0.08 & 0.04 & 0.04 & 0.06 & 0.04 \\
\hline Total & 99.82 & \begin{tabular}{|l|}
100.87 \\
\end{tabular} & 101.83 & 100.50 & 101.07 & 100.63 & 101.54 & 101.09 & 100.09 & 100.86 & 100.55 \\
\hline Cations for & 2 oxygens & & & & & & & & & & \\
\hline $\mathrm{Si}$ & 12.49 & 12.29 & 12.19 & 12.06 & 12.08 & 11.46 & 11.40 & 12.08 & 12.08 & 11.95 & 11.90 \\
\hline $\mathrm{Al}$ & 3.55 & 3.66 & 3.89 & 3.60 & 3.94 & 4.53 & 4.57 & 3.96 & 4.05 & 4.05 & 4.07 \\
\hline $\mathrm{Fe}$ & 0.06 & 0.09 & 0.02 & 0.03 & 0.02 & 0.01 & 0.00 & 0.02 & 0.06 & 0.01 & 0.03 \\
\hline $\mathrm{Mn}$ & 0.00 & 0.00 & 0.00 & 0.00 & 0.00 & 0.00 & 0.00 & 0.00 & 0.00 & 0.00 & 0.00 \\
\hline $\mathrm{Mg}$ & 0.04 & 0.12 & 0.00 & 0.02 & 0.00 & 0.00 & 0.00 & 0.00 & 0.03 & 0.00 & 0.00 \\
\hline $\mathrm{Ca}$ & 0.05 & 0.08 & 0.03 & 0.12 & 0.07 & 0.65 & 0.73 & 0.08 & 0.10 & 0.16 & 0.16 \\
\hline $\mathrm{Na}$ & 3.05 & 3.24 & 3.42 & 3.49 & 3.66 & 3.23 & 3.20 & 3.57 & 3.15 & 3.70 & 3.81 \\
\hline $\mathrm{K}$ & 0.02 & 0.01 & 0.01 & 0.01 & 0.01 & 0.02 & 0.02 & 0.01 & 0.01 & 0.01 & 0.01 \\
\hline Total & 19.26 & 19.50 & 19.57 & 19.61 & 19.79 & 19.90 & 19.92 & 19.73 & 19.47 & 19.89 & 19.98 \\
\hline $\mathrm{Ab} \%$ & 97.82 & 97.23 & 98.70 & 96.29 & 97.75 & 82.93 & 81.02 & 97.43 & 96.66 & 95.49 & 95.69 \\
\hline An $\%$ & 1.70 & 2.39 & 0.95 & 3.39 & 1.99 & 16.68 & 18.55 & 2.31 & 3.09 & 4.19 & 4.08 \\
\hline Or $\%$ & 0.48 & 0.38 & 0.36 & 0.32 & 0.26 & 0.40 & 0.43 & 0.26 & 0.26 & 0.32 & 0.22 \\
\hline
\end{tabular}

\# Number of analysed spots

The analyzed samples contain relatively high $\mathrm{SiO}_{2}$ (48$53 \%$ ). They plot in the field of basalt in $\mathrm{Ga}$ vs. $\mathrm{Zr} / \mathrm{TiO}_{2}$ diagram (Fig. 4a). Four of the samples plot in the field of sub-alkaline basalt and one sample (Sample no. 163) plot at the boundary line of sub-alkaline and alkaline basalt in $\mathrm{Zr} / \mathrm{TiO}_{2}$ vs. Nb/Y diagram (Fig. 4b) of Winchester and Floyd (1977). High $\mathrm{FeO} / \mathrm{MgO}$ ratios (>1.4) indicate their tholeiitic nature.

The effect of whole rock chemistry on metamorphic amphibole was assessed by plots of whole rock composition vs. average composition of matrix amphiboles. $\mathrm{MgO} /(\mathrm{FeO}+\mathrm{MgO})$ in whole rock and amphiboles display linear positive correlation $\left(\mathrm{R}^{2}=0.83\right.$ (Fig. 5a). However, $\mathrm{TiO}_{2}, \mathrm{Na}_{2} \mathrm{O}$ and $\mathrm{Al}_{2} \mathrm{O}_{3}$ show no systematic relations between whole rock and matrix amphiboles (Figs. 5b, 5c and 5d).

Positive correlation of $\mathrm{MgO} /(\mathrm{FeO}+\mathrm{MgO})$ between whole rock and amphiboles is also observed in other metamorphic belts (Zenk and Schulz 2004). It is believed that chemistry of metamorphic amphiboles at the onset of crystallization under greenschist-facies condition may be partly affected by whole rock chemistry, but at higher metamorphic grade it is controlled principally by pressure and temperature (Graham 1974; Liard and Albee 1981; Zenk and Schulz 2004). Further, in the present case, all rocks contain nearly constant assemblage of amphiboles + chlorite + epidote + plagioclase + quartz $+\mathrm{Fe}-\mathrm{Ti}$ oxides . As the individual minerals are buffered by the assemblage, variation in mineral chemistry within and among samples can be related to differences in metamorphic grade or facies series rather than bulk rock chemistry (Liard 1980; Liard and Albee 1981). Presence of amphiboles with variable composition within a single grain also entails for the pressure-temperature control on amphibole composition.

\section{MINERAL CHEMISTRY}

\section{Analytical procedures}

JEOL Superprobe 733 (Hokkaido University) electronprobe microanalyser was used for spot (1-5 $\mu \mathrm{m})$ analyses of amphiboles and plagioclases. Analyses were performed with $15 \mathrm{kV}$ accelerating voltage, and $12 \mathrm{nA}$ beam current. Natural and synthetic silicates and oxides were used for standards and conventional ZAF method was employed for matrix correction. Analyses were made in three thinsections from each sample. 
More than 250 spot analyses were carried out on amphiboles alone, out of which 180 analyses are used for interpretation. Zoned porphyroblasts were analyzed at cores (pale green inner part) and rims (dark green outer part). Matrix amphiboles do not show chemical zoning and, therefore, they were analyzed only at cores. About 75 grains of relic (porphyroclast) and recrystallized (porphyroblasts and matrix) plagioclase coexisting with amphiboles were analyzed at cores and rims.

\section{Plagioclase composition}

The average plagioclase compositions are presented in Table 2. Plagioclase are mainly albite $(\mathrm{An}<10 \%)$ except for Sample no. 163 which contains oligoclase $(A n=17-$ $19 \%$ ). Only a few plagioclase grains are slightly zoned. Majority of the zoned grains show slight increase in Ancontent towards rim. An content of plagioclase is $<2 \%$ in the chlorite zone samples, $2-7 \%$ in the biotite zone samples and up to $19 \%$ in the garnet zone samples.

Table 3: Average compositions of amphiboles in metabsites, Cation normalization according to Schumacher (1997). $X \mathrm{Mg}=\mathrm{Mg} /$ $\left(\mathrm{Fe}^{2+}+\mathrm{Mg}\right)$

\begin{tabular}{|c|c|c|c|c|c|c|c|c|c|c|c|c|c|}
\hline \multirow{3}{*}{$\begin{array}{c}\text { S. No. } \\
\text { Grain type } \\
\text { Position }\end{array}$} & \multirow{3}{*}{$\begin{array}{c}42 \\
\text { Matrix } \\
\text { Core }\end{array}$} & \multicolumn{3}{|c|}{623} & \multicolumn{3}{|c|}{163} & \multicolumn{3}{|c|}{625} & \multicolumn{3}{|c|}{180} \\
\hline & & \multicolumn{2}{|c|}{ Porphyroblast } & \multirow{2}{*}{$\begin{array}{c}\text { Matrix } \\
\text { Core }\end{array}$} & \multicolumn{2}{|c|}{ Porphyroblast } & \multirow{2}{*}{$\begin{array}{c}\text { Matrix } \\
\text { Core }\end{array}$} & \multicolumn{2}{|c|}{ Porphyroblast } & \multirow{2}{*}{$\begin{array}{c}\text { Matrix } \\
\text { Core }\end{array}$} & \multicolumn{2}{|c|}{ Porphyroblast } & \multirow{2}{*}{$\begin{array}{c}\text { Matrix } \\
\text { Core }\end{array}$} \\
\hline & & Core & Rim & & Core & Rim & & Core & Rim & & Core & Rim & \\
\hline \# & 8 & 13 & 14 & 11 & 27 & 12 & 30 & 9 & 13 & 13 & 8 & 13 & 9 \\
\hline $\mathrm{SiO}_{2}$ & 54.94 & 51.89 & 44.11 & 45.85 & 48.65 & 42.32 & 42.31 & 49.73 & 42.66 & 42.93 & 49.02 & 41.77 & 41.56 \\
\hline $\mathrm{TiO}_{2}$ & 0.05 & 0.11 & 0.33 & 0.28 & 0.50 & 0.48 & 0.41 & 0.14 & 0.31 & 0.31 & 0.32 & 0.39 & 0.48 \\
\hline $\mathrm{Al}_{2} \mathrm{O}_{3}$ & 1.47 & 5.12 & 14.47 & 12.51 & 6.74 & 14.44 & 14.74 & 5.82 & 13.15 & 13.00 & 6.60 & 13.36 & 13.62 \\
\hline $\mathrm{FeO}^{*}$ & 11.75 & 12.47 & 14.82 & 14.56 & 18.89 & 20.16 & 19.93 & 16.94 & 19.44 & 19.22 & 19.32 & 21.85 & 21.73 \\
\hline $\mathrm{MnO}$ & 0.23 & 0.23 & 0.28 & 0.27 & 0.31 & 0.29 & 0.28 & 0.24 & 0.22 & 0.21 & 0.32 & 0.33 & 0.31 \\
\hline $\mathrm{MgO}$ & 16.15 & 14.81 & 9.76 & 10.77 & 10.35 & 6.74 & 6.63 & 12.91 & 8.56 & 8.70 & 9.93 & 6.25 & 6.17 \\
\hline $\mathrm{CaO}$ & 12.31 & 12.23 & 11.69 & 11.80 & 11.53 & 11.31 & 11.10 & 10.73 & 10.66 & 10.72 & 11.26 & 11.06 & 10.91 \\
\hline $\mathrm{Na}_{2} \mathrm{O}$ & 0.29 & 0.57 & 1.52 & 1.33 & 0.93 & 1.75 & 1.83 & 0.94 & 2.12 & 2.07 & 0.84 & 1.59 & 1.74 \\
\hline $\mathrm{K}_{2} \mathrm{O}$ & 0.00 & 0.02 & 0.23 & 0.19 & 0.05 & 0.26 & 0.33 & 0.00 & 0.12 & 0.11 & 0.04 & 0.39 & 0.47 \\
\hline Total & 97.20 & 97.45 & 97.20 & 97.56 & 97.95 & 97.76 & 97.55 & 97.48 & 97.25 & 97.27 & 97.65 & 96.99 & 97.00 \\
\hline \multicolumn{14}{|c|}{ Cations for 23 Oxygens (recalculated on the basis of $13 \mathrm{eCNK}$ ) } \\
\hline $\mathrm{Si}$ & 7.86 & 7.43 & 6.47 & 6.68 & 7.15 & 6.33 & 6.34 & 7.15 & 6.33 & 6.36 & 7.22 & 6.34 & 6.31 \\
\hline $\mathrm{Al}^{\mathrm{IV}}$ & 0.14 & 0.57 & 1.53 & 1.32 & 0.85 & 1.67 & 1.66 & 0.85 & 1.67 & 1.64 & 0.78 & 1.66 & 1.69 \\
\hline Sum $T$ & 8.00 & 8.00 & 8.00 & 8.00 & 8.00 & 8.00 & 8.00 & 8.00 & 8.00 & 8.00 & 8.00 & 8.00 & 8.00 \\
\hline $\mathrm{Al}^{\mathrm{VI}}$ & 0.11 & 0.30 & 0.97 & 0.82 & 0.32 & 0.88 & 0.94 & 0.15 & 0.63 & 0.63 & 0.36 & 0.73 & 0.75 \\
\hline $\mathrm{Ti}$ & 0.01 & 0.01 & 0.04 & 0.03 & 0.05 & 0.05 & 0.05 & 0.02 & 0.03 & 0.03 & 0.04 & 0.04 & 0.05 \\
\hline $\mathrm{Fe}^{3+}$ & 0.15 & 0.32 & 0.33 & 0.34 & 0.52 & 0.49 & 0.47 & 1.10 & 0.95 & 0.91 & 0.55 & 0.71 & 0.68 \\
\hline $\mathrm{Fe}^{2+}$ & 1.25 & 1.18 & 1.49 & 1.43 & 1.81 & 2.03 & 2.03 & 0.95 & 1.47 & 1.47 & 1.84 & 2.07 & 2.08 \\
\hline $\mathrm{Mg}$ & 3.45 & 3.16 & 2.14 & 2.34 & 2.27 & 1.50 & 1.48 & 2.77 & 1.89 & 1.92 & 2.18 & 1.41 & 1.40 \\
\hline $\mathrm{Mn}$ & 0.03 & 0.03 & 0.03 & 0.03 & 0.04 & 0.04 & 0.04 & 0.03 & 0.03 & 0.03 & 0.04 & 0.04 & 0.04 \\
\hline Sum $M 1$ & 5.00 & 5.00 & 5.00 & 5.00 & 5.00 & 5.00 & 5.00 & 5.00 & 5.00 & 5.00 & 5.00 & 5.00 & 5.00 \\
\hline $\mathrm{Ca}$ & 1.89 & 1.88 & 1.84 & 1.84 & 1.82 & 1.81 & 1.78 & 1.66 & 1.70 & 1.70 & 1.78 & 1.80 & 1.78 \\
\hline $\mathrm{Na}_{\mathrm{M} 4}$ & 0.07 & 0.12 & 0.16 & 0.16 & 0.18 & 0.19 & 0.22 & 0.26 & 0.30 & 0.30 & 0.22 & 0.20 & 0.22 \\
\hline Sum $M 4$ & 1.96 & 2.00 & 2.00 & 2.00 & 2.00 & 2.00 & 2.00 & 1.91 & 2.00 & 2.00 & 2.00 & 2.00 & 2.00 \\
\hline $\mathrm{Na}_{\mathrm{A}}$ & 0.00 & 0.04 & 0.27 & 0.22 & 0.08 & 0.32 & 0.31 & 0.00 & 0.30 & 0.30 & 0.02 & 0.27 & 0.29 \\
\hline K & 0.00 & 0.00 & 0.04 & 0.04 & 0.01 & 0.05 & 0.06 & 0.00 & 0.02 & 0.02 & 0.01 & 0.08 & 0.09 \\
\hline $\operatorname{Sum} A$ & 0.00 & 0.04 & 0.31 & 0.25 & 0.09 & 0.37 & 0.38 & 0.00 & 0.33 & 0.32 & 0.03 & 0.34 & 0.38 \\
\hline$X \mathrm{Mg}$ & 0.73 & 0.73 & 0.59 & 0.62 & 0.56 & 0.43 & 0.42 & 0.75 & 0.56 & 0.57 & 0.54 & 0.41 & 0.40 \\
\hline $\mathrm{Ti} / \mathrm{Al}$ & 0.02 & 0.01 & 0.01 & 0.01 & 0.05 & 0.02 & 0.02 & 0.02 & 0.02 & 0.02 & 0.03 & 0.02 & 0.02 \\
\hline
\end{tabular}

\# Number of amphibole grains analysed, Oxides are in wt\% 
(a) Chlorite zone (No. 42)

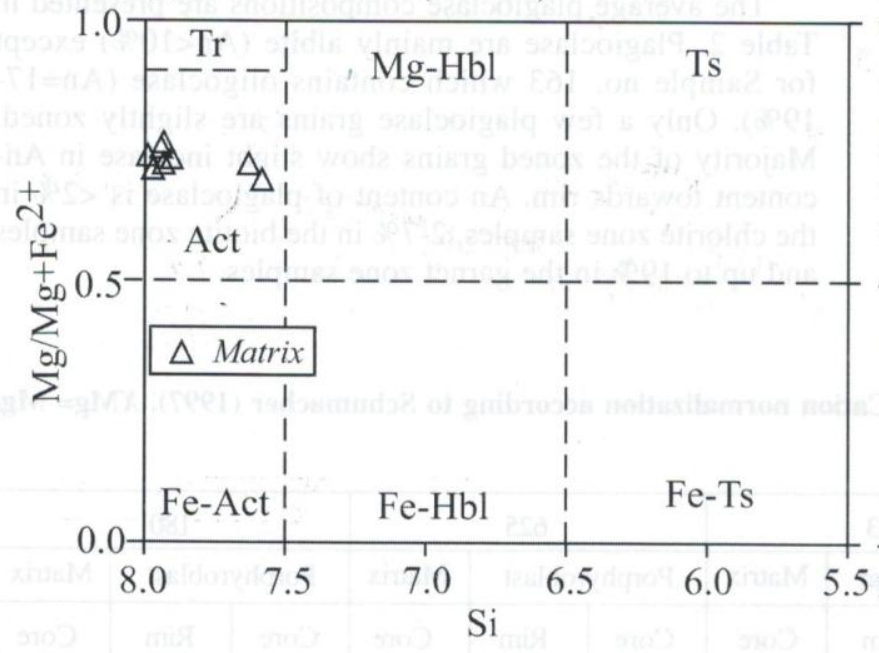

(c) Garnet zone (No.163)

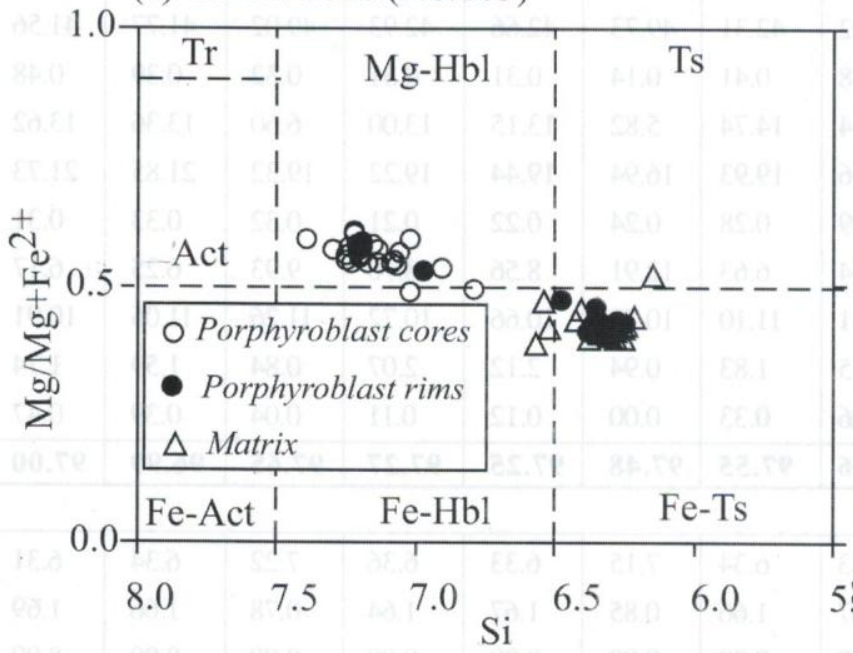

(e) Garnet zone (No. 180)

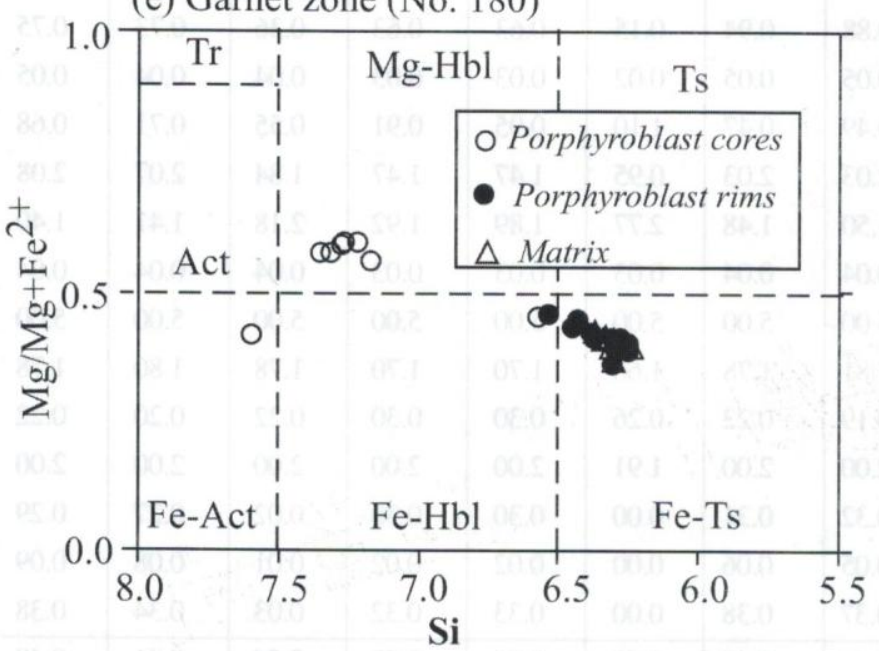

Fig. 6: Classification of Ca-amphiboles according to Leake et al. (1997). The amphibole porphyroblasts show clear compositional zoning in all the samples. Tr: tremolite, Act: actinolite, Mg-Hbl: magnesio-hornblende, Ts: tschermakite, Fe-Act: ferro-actinolite, Fe-Hbl: ferro-hornblende, Fe-Ts: ferro-tschermakite. Cataion values are in per formula unit. (b) Biotite zone (No.623)

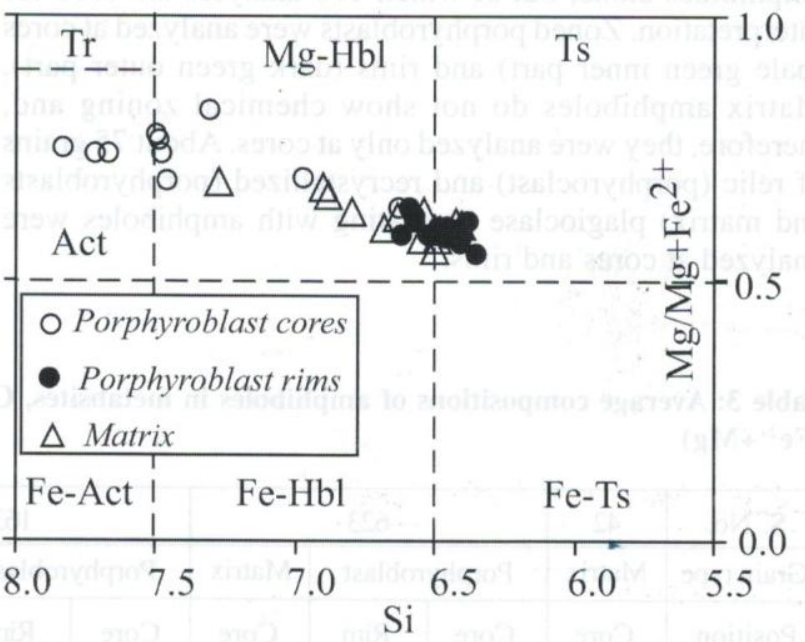

(d) Garnet zone (No.625

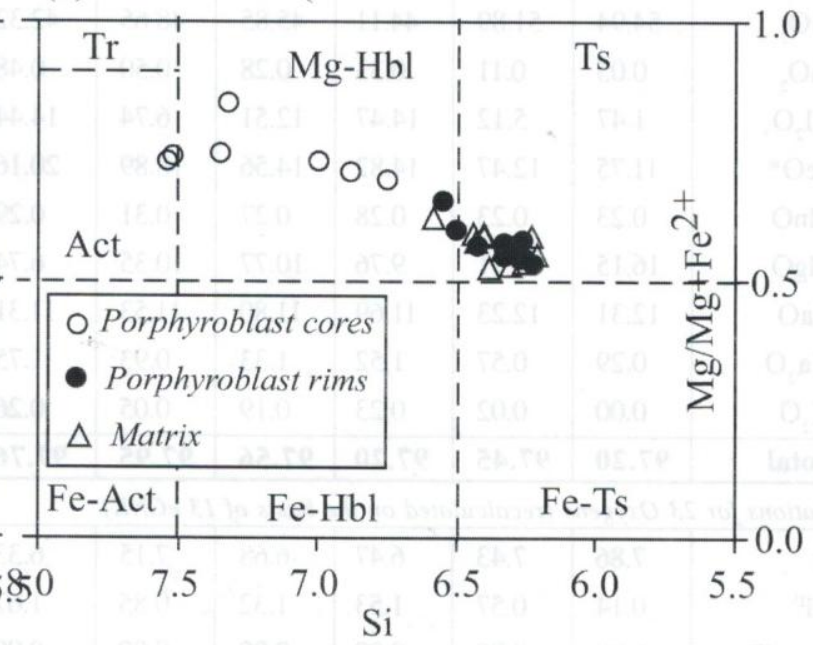




\section{Amphibole composition}

The amphibole analyses were first recalculated on the basis of 23 oxygens assuming all $\mathrm{Fe}$ as $\mathrm{Fe}^{2+}$. Crystallographic site assignments, cation recalculation, and calculation of $\mathrm{Fe}^{3+}$ was performed according to the procedures given in Schumacher (1997) assuming total cation $=13$ excluding $\mathrm{Ca}, \mathrm{Na}$, and $\mathrm{K}$ ( 13 eCNK method). In metamorphic amphiboles, $\mathrm{Al}^{\mathrm{IV}}, \mathrm{Al}{ }^{\mathrm{VI}}, \mathrm{Ti},\left(\mathrm{Al}^{\mathrm{VI}}+\mathrm{Fe}^{3+}+\mathrm{Ti}\right), \mathrm{Na}_{\mathrm{M} 4}$, $\mathrm{Na}_{A}$, and $\left(\mathrm{Na}_{A}+\mathrm{K}\right)$ increase and $\mathrm{Si}$ and $X \mathrm{Mg}\left(\mathrm{Mg} / \mathrm{Mg}+\mathrm{Fe}^{2+}\right)$ decrease with increasing metamorphic grade (Liard and Albee 1981). Therefore, the contents of above cations (per formula unit) are used for discussions. Complete analytical data set are given in Paudel et al. (2005). The average amphibole compositions are presented in Table 3.

\section{Amphibole classification}

Amphibole classification was followed after Leake et al. (1997). The amphiboles in most of the samples contain $\left(\mathrm{Ca}+\mathrm{Na}_{M 4}\right)$ e" 1.0 and $\mathrm{Na}_{M 4}<0.5$ (Table 3 ), suggesting all of them to belong to the calcic-amphibole group. Amphibole analyses of each sample are shown on $\mathrm{Si}-\mathrm{XMg}$ diagram for further classification (Fig. 6).

The matrix amphiboles of the chlorite zone sample (Sample no. 42) are actinolites with average $\mathrm{Si}$ and $\mathrm{XMg}$ values of 7.86 and 0.73 , respectively (Table 3, Fig. 6a). In the biotite zone (Sample no. 623), the porphyroblast cores range in composition from actinolite to magnesiohornblende with average $\mathrm{Si}$ of 7.43 and average $\mathrm{XMg}$ of 0.73 . The rims of porphyroblast and matrix amphiboles range in composition from magnesio-hornblende to tschermakite (Fig. 6b). Average $\mathrm{Si}$ in the porphyroblast rims and matrix amphiboles are 6.47 and 6.68 , and average $X \mathrm{Mg}$ are 0.59 and 0.56 , respectively.

In the garnet zone, porphyroblast cores in all three samples belong to the magnesio-hornblende. The porphyroblast rims and matrix amphiboles in Sample no. 163 plot in ferro-tschermakite field, those in Sample no. 625 plot in tschermakite field, and those in Sample no. 180 plot in ferro-tschermakite field (Figs. 6c, 6d and 6e). The average $\mathrm{Si}$ in the cores ranges from 7.15 to 7.22 and that in the rims ranges from 6.31 to 6.36 (Table 3). The porphyroblast rims and matrix amphiboles in Sample nos. 163 and 180 are $X \mathrm{Mg}$-poor (0.40-0.43) compared to those in Sample no. $625(X \mathrm{Mg}=0.56-0.57)$.

\section{Zoning in porphyroblasts}

Compositional zoning of amphibole porphyroblasts is clear as shown in Fig. 6. In Ca-amphiboles, Si decreases and $\mathrm{Al}^{\mathrm{IV}}$ and $\mathrm{Ti}$ increase with increasing temperature; and $\mathrm{Al}^{\mathrm{VI}}$ and $\mathrm{Na}_{M 4}$ increase with increasing pressure (Raase 1974; Brown 1977, Holland and Richardson 1979; Spear 1980). Core and rim compositions of porphyroblasts from all samples are shown on $\mathrm{Al}^{\mathrm{IV}}$ vs. $\mathrm{Na}_{M 4}$ and $\mathrm{Al}^{\mathrm{IV}}$ vs. $\mathrm{Al}^{\mathrm{VI}}$ diagrams (Figs. 7a and 7b). The core and rim compositions plot in distinctly different fields in both the diagrams. The $\mathrm{Al}^{\mathrm{IV}}$ for cores ranges from 0.17 to 1.43 and for the rims ranges from 1.38 to 1.71 . The $\mathrm{Na}_{M 4}$ of cores is in between 0.04 and 0.22 and that of the rims is in between 0.09 and 0.35 . The $\mathrm{Al}^{\mathrm{IV}}$ ranges from 0.0 to 0.63 in the cores 0.43 to 1.08 in the rims. These compositional data clearly indicate the growth of cores and rims at different metamorphic grade and P-T conditions.

Nature of chemical zoning of porphyroblast was further checked by detail chemical profiling (15-20 point analyses) of two optically zoned grains in Sample nos. 163 and 625 (Figs. 7c and 7d). In relatively large $(\sim 0.8 \mathrm{~mm})$ grain of less-sheared sample (Sample no. 163), the core is relatively homogeneous, and zoning is confined only to the outer part of the grain (Fig. 7c). In relatively small $(\sim 0.2$ $\mathrm{mm}$ ) grain of highly sheared sample (Sample no. 625), the zoning is continuous from core to rim (Fig. 7d). In both the cases, $\mathrm{Al}^{\mathrm{IV}},\left(\mathrm{Al}^{\mathrm{VI}}+\mathrm{Fe}^{3+}+\mathrm{Ti}\right)$, and $\left(\mathrm{Na}_{A}+\mathrm{K}\right)$ increase from core to rim. In the margin of the grains, the values for all above cations decrease again. The Si decreases from cores to rims and finally slightly increases at the margin. The profiles show prograde metamorphic zonation from core to rim, and retrograde re-equilibration at the margin. This zoning pattern is similar to the compositional zoning in garnets in associated metapelites (see Fig. 8 of Paudel and Arita 2000).

\section{Compositional variation of syn-kinematic amphiboles in matrix}

Liard and Albee (1981) proposed several cation-based diagrams for $\mathrm{Ca}$-amphiboles that are capable of separating amphiboles from different metapelitic metamorphic zones. Compositions of syn-kinematic amphiboles in matrix from the present area are plotted in some of those diagrams (Fig. $8)$. The amphiboles from the chlorite, biotite, and garnet zone samples plot in different fields in $\mathrm{Al}^{\mathrm{IV}}$ vs. Ti (Fig. 8a), $\mathrm{Al}^{\mathrm{IV}}$ vs. $\mathrm{Al}^{\mathrm{VI}}$ (Fig. 8 b), $\left(\mathrm{Al}^{\mathrm{VI}}+\mathrm{Fe}^{3+}+\mathrm{Ti}\right)$ vs. $\mathrm{Na}_{M 4}(\mathrm{Fig} .8 \mathrm{c})$ and $\left(\mathrm{Al}^{\mathrm{VI}}+\mathrm{Fe}^{3+}+\mathrm{Ti}\right)$ vs. $\mathrm{Na}_{4}$ (Fig. 8d) diagrams. General increase of $\mathrm{Al}^{\mathrm{IV}}, \mathrm{Ti},\left(\mathrm{Al}^{\mathrm{VI}}+\mathrm{Fe}^{3+}+\mathrm{Ti}\right), \mathrm{Na}_{M 4}$ and $\mathrm{Na}_{A}$ from the chlorite zone to biotite zone, and then to garnet zone is quite clear from the diagrams as well as from the average values in Table 3.

The average $\mathrm{Al}^{\mathrm{IV}}$ in the chlorite zone is 0.14 , in the biotite zone is 1.32 and in the garnet zone is 1.64-1.69 (averages of three samples, Table 3 ). Similarly, average Ti increases from 0.01 in the chlorite zone to 0.03 in the biotite zone, and (0.03-0.05) in the garnet zone. The $\left(\mathrm{Al}^{\mathrm{VI}}+\mathrm{Fe}^{3+}+\mathrm{Ti}\right)$ content varies from 0.27 in the chlorite zone to 1.0 in the biotite zone, and (1.46-1.58) in the garnet zone. The $\mathrm{Na}_{M 4}$ varies from 0.07 in the chlorite zone to 0.16 in the biotite zone and to $(0.22-0.30)$ in the garnet zone. The $\mathrm{Al}^{\mathrm{VI}}$ increases from 0.11 in the chlorite zone to 0.82 in the biotite zone. It does not vary much between the biotite (0.82) and garnet zones (0.63-0.94). 
(a)

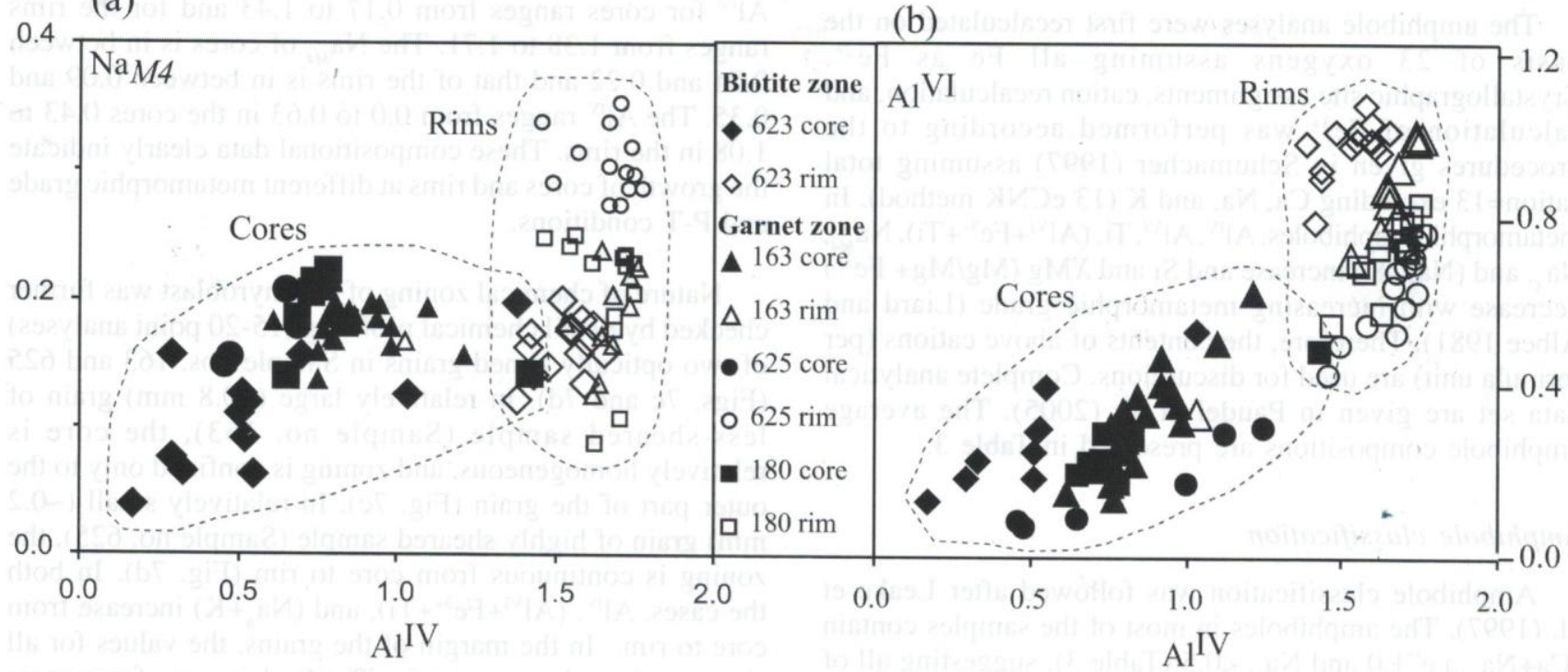

(c)
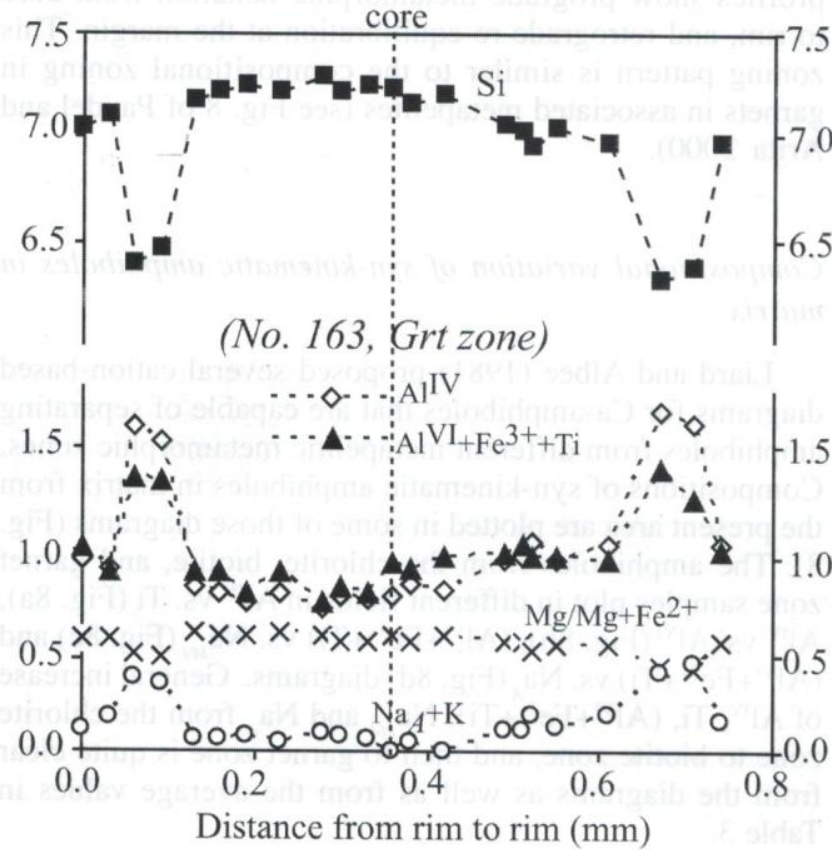

(d)

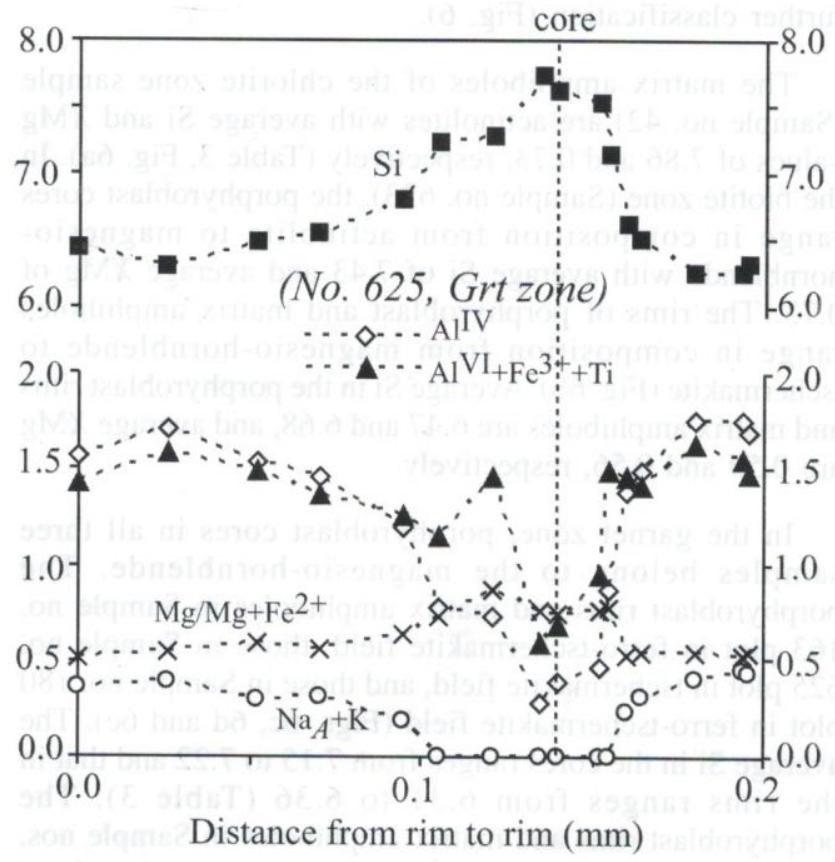

Fig. 7: (a) and (b) Chemical compositions of cores and rims of amphibole porphyroblasts in the MCT zone. Cores and rims plot in distinctly different fields in $\mathrm{Al}^{\mathrm{IV}}$ vs. $\mathrm{Na}_{M 4}$ and $\mathrm{Al}^{\mathrm{VI}}$ plots, indicating different metamorphic conditions of recrystallization of cores and rims. (c) Compositional profile of an amphibole porphyroblast in No. 163. Relatively large porphyroblast shows almost uniform composition in the cores and zoning only in the rims and margins. (d) Compositional profile of a porphyroblast in No. 625. Relatively small porphyroblasts show almost continuous zonation from core to rim. Cation values are in per formula unit. 
(a)

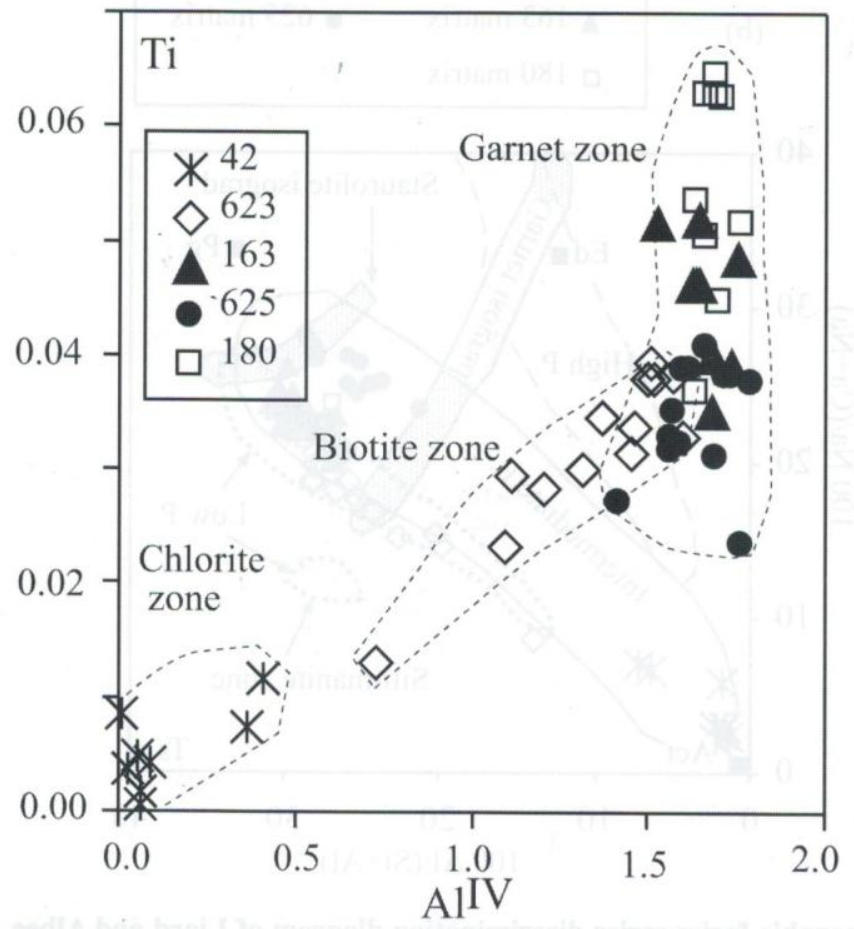

(c)

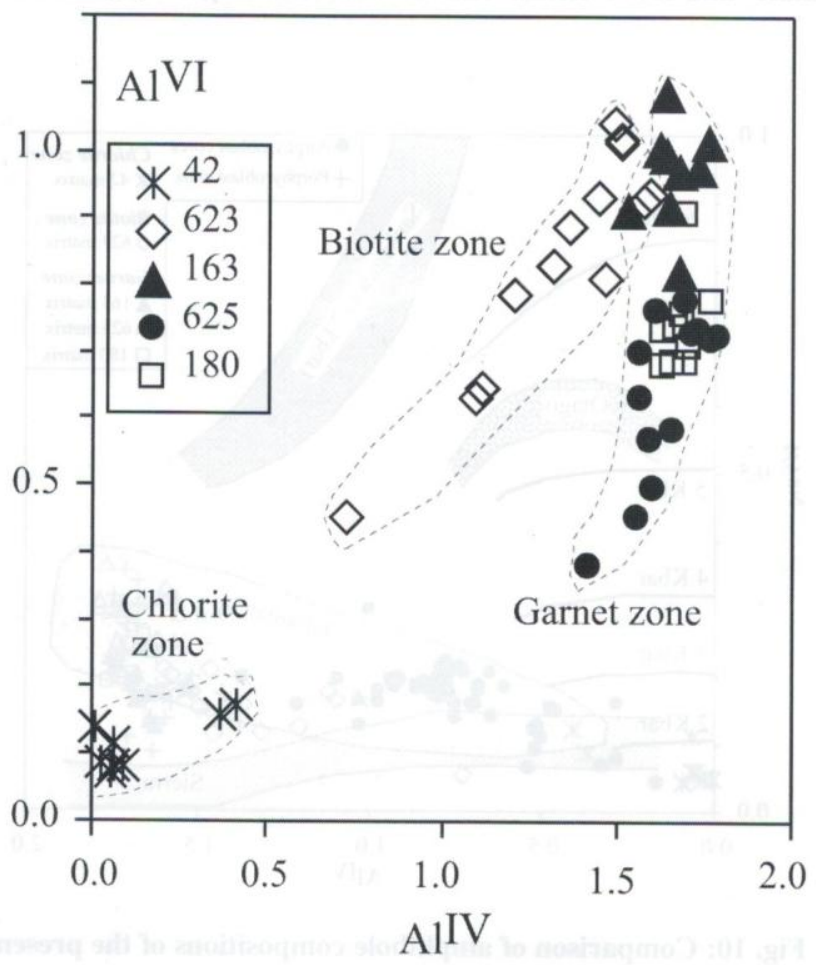

(b)

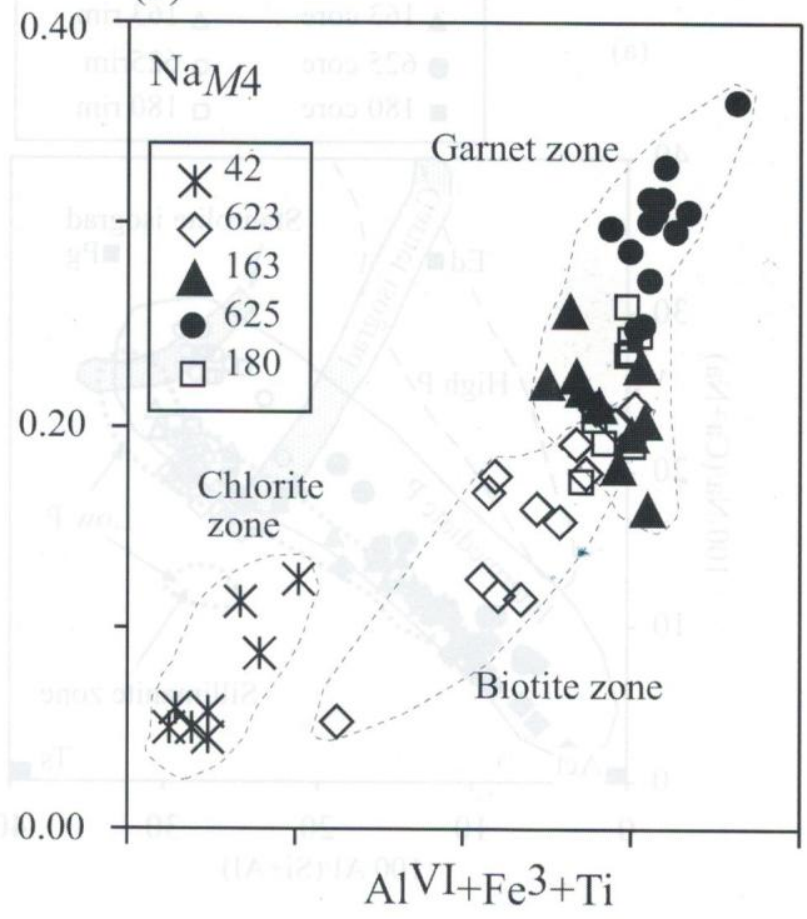

(d)

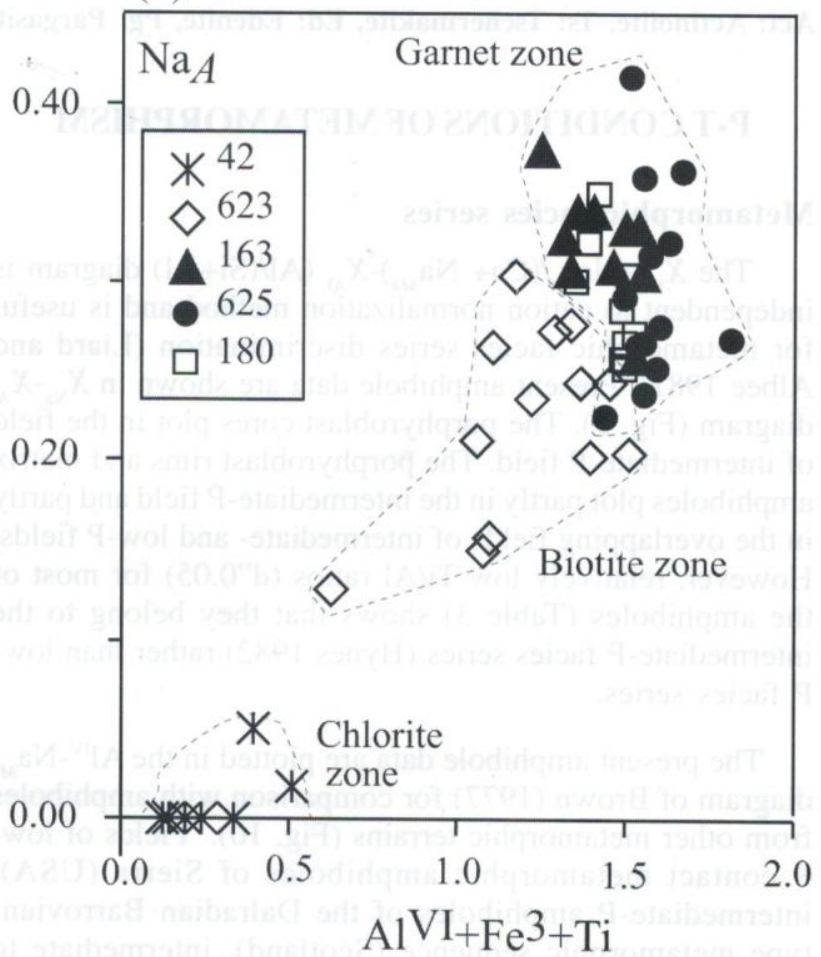

Fig. 8: Compositional variation in syn-kinematic matrix amphiboles. Matrix amphible compositions from the chlorite, biotite and garnet zone samples plot in different fields in the various cation-based diagrams. Cation values are in per formula unit. 

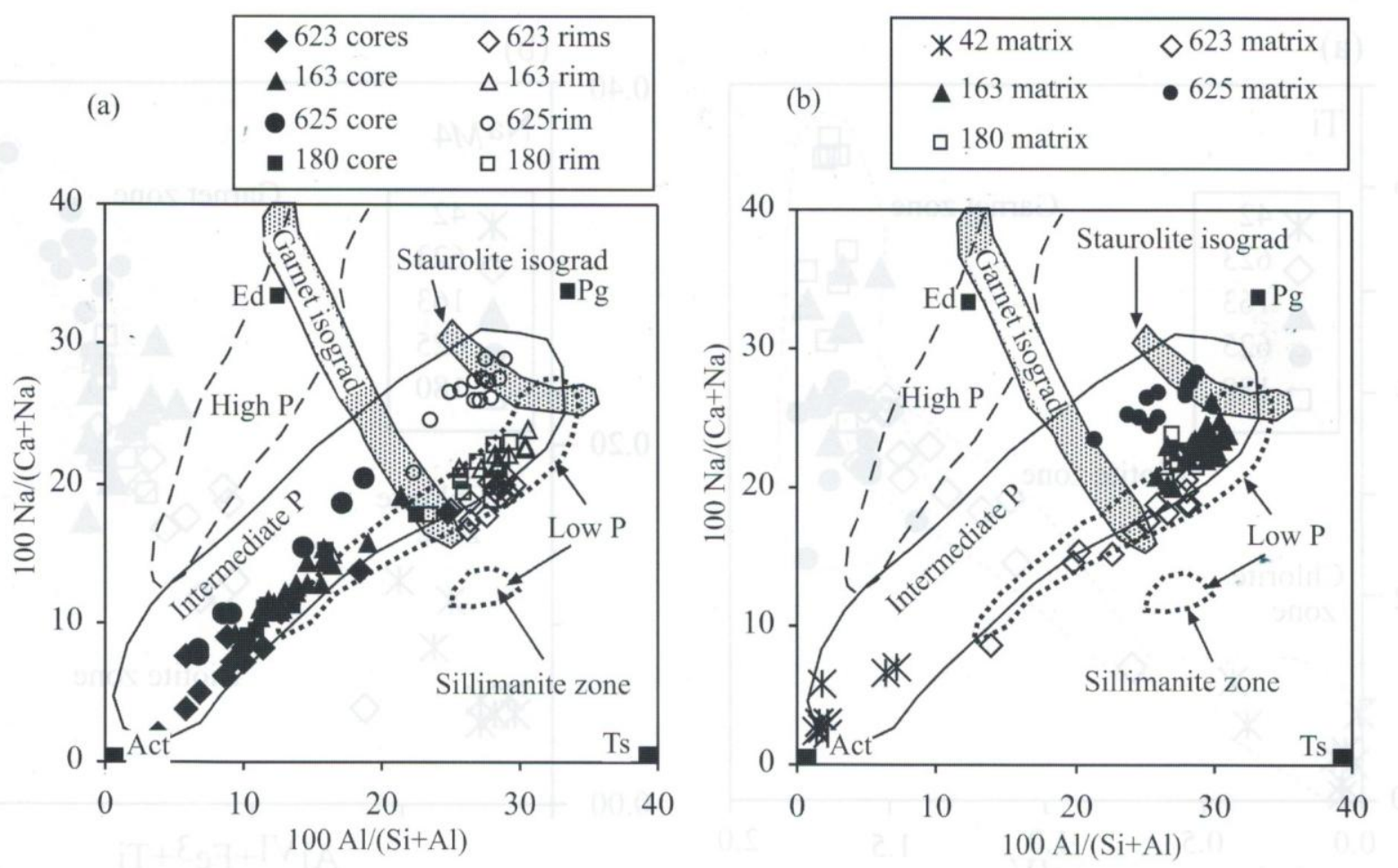

Fig. 9: Porphyroblasts (a) and matrix (b) compositions in metamorphic facies series discrimination diagram of Liard and Albee (1981). Most of the data plot in the overlapping fields of intermediate- and low-P fields. Cation values are in per formula unit. Act: Actinolite, Ts: Tschermakite, Ed: Edenite, Pg: Pargasite.

\section{P-T CONDITIONS OF METAMORPHISM}

\section{Metamorphic facies series}

The $X_{\mathrm{Na}}\left(\mathrm{Na}_{M 4} /\left(\mathrm{Ca}+\mathrm{Na}_{M 4}\right)-X_{\mathrm{Al}}(\mathrm{Al} /(\mathrm{Si}+\mathrm{Al})\right.$ diagram is independent to cation normalization method and is useful for metamorphic facies series discrimination (Liard and Albee 1981). Present amphibole data are shown in $X_{\mathrm{Na}}-X_{\mathrm{AI}}$ diagram (Fig. 9). The porphyroblast cores plot in the field of intermediate-P field. The porphyroblast rims and matrix amphiboles plot partly in the intermediate-P field and partly in the overlapping fields of intermediate- and low-P fields. However, relatively low $\mathrm{Ti} / \mathrm{Al}$ ratios (d" 0.05 ) for most of the amphiboles (Table 3) shows that they belong to the intermediate-P facies series (Hynes 1982) rather than low$\mathrm{P}$ facies series.

The present amphibole data are plotted in the $\mathrm{Al}^{\mathrm{IV}}-\mathrm{Na}_{M 4}$ diagram of Brown (1977) for comparison with amphiboles from other metamorphic terrains (Fig. 10). Fields of low$\mathrm{P}$ contact metamorphic amphiboles of Sierra (USA), intermediate-P amphiboles of the Dalradian Barroviantype metamorphic sequence (Scotland), intermediate to high-P amphiboles of Otago (Newzeland), and high-P metamorphic amphiboles of Sanbagawa (Japan) are shown in the figure. The present data overlap with the Dalradian amphiboles, confirming intermediate-P Barrovian-type metamorphism.

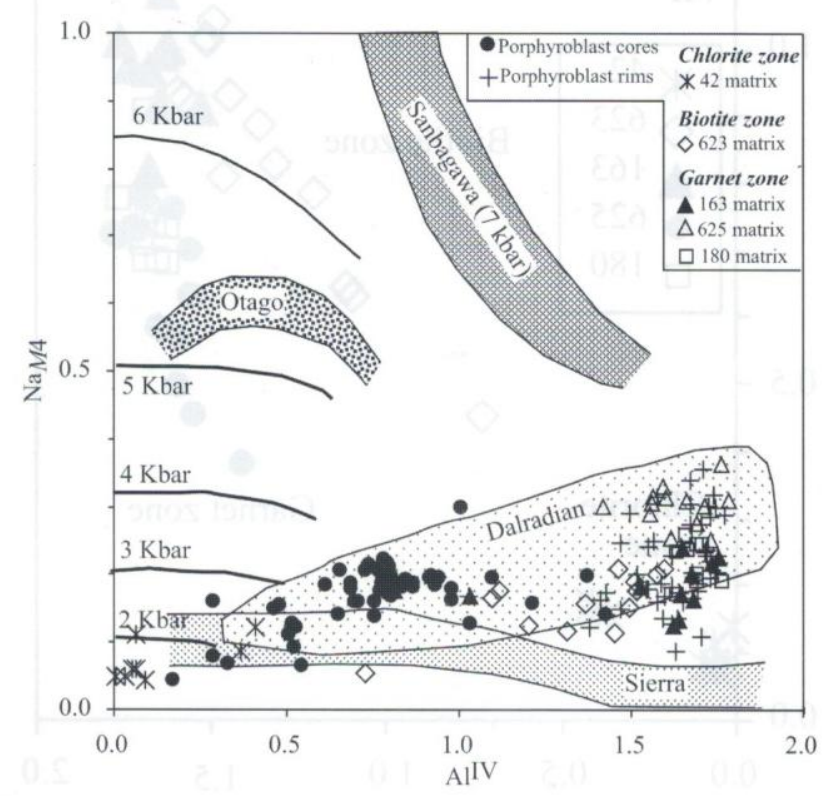

Fig. 10: Comparison of amphibole compositions of the present study with those from other metamorphic terrains. Compositionl fields for Sierra (USA), Otago (Newzealand) and Sanbagawa (Japan) amphiboles and qualitative pressure grids from Brown (1977). Field for the Dalradian amphiboles delineated from the amphibole compositional data of Zenk and Schulz (2004). Cation values are in per formula unit. 
Table 4: P-T estimation using amphibole-plagioclase thermometry of Holland and Blundy (1994) and empirical thermobarometry of Gerya et al. (1997) revised by Zenk and Schulz (2004). Average compositional data of amphiboles and coexisting plagioclase were used for calculation. The results are affected by minimum error of $\pm 50^{\circ} \mathrm{C} / 1.2 \mathrm{kbar}$. MCT: Main Central Thrust, TS II: Thrust Sheet II, Grt: Garnet, Bt: Biotite

\begin{tabular}{|l|c|c|c|c|c|c|c|}
\hline Tectonic Unit & $\begin{array}{c}\text { Sample } \\
\text { No. }\end{array}$ & $\begin{array}{c}\text { Core } \\
\left(\mathrm{T}{ }^{\circ} \mathrm{C}\right)\end{array}$ & $\begin{array}{c}\text { Rim } \\
\left(\mathrm{T}^{\circ} \mathrm{C}\right)\end{array}$ & $\begin{array}{c}\text { Matrix } \\
\left(\mathrm{T}{ }^{\circ} \mathrm{C}\right)\end{array}$ & $\begin{array}{c}\text { Core } \\
(\mathrm{P} \text { kbar })\end{array}$ & $\begin{array}{c}\text { Rim } \\
(\mathrm{P} \text { kbar })\end{array}$ & $\begin{array}{c}\text { Matrix } \\
(\mathrm{P} \text { kbar })\end{array}$ \\
\hline MCT zone (Grt zone) & $180^{*}$ & 560 & 630 & 620 & & & \\
& $625^{*}$ & - & 635 & 635 & & & \\
& $163^{\S}$ & 505 & 625 & 625 & 3.2 & 5.9 & 5.9 \\
\hline TS II (Bt zone) & $163^{*}$ & 570 & 640 & 610 & & & \\
\hline
\end{tabular}

* $\mathrm{Hbl}$-Pl thermometry of Holland and Blundy (1994)

\$Thermobarometry of Gerya et al. (1997) modified by Zenk and Schulz (2004)

\section{P-T evolution}

One sample (Sample no. 163) from the study area contains plagioclase with $\mathrm{An}>10 \%$ and is suitable for application of the revised version (revised by Zenk and Schulz 2004) of the empirical amphibole thermobarometer of Gerya et al. (1997) for P-T calculation. Application of this thermobarometer to Sample no. 163 yields average temperature of $\sim 505^{\circ} \mathrm{C}$ at pressure of $3.2 \mathrm{kbar}$ for porphyroblast cores. For porphyroblast rims and matrix amphiboles, peak temperatures are calculated to be $\sim 625^{\circ} \mathrm{C}$ at 5.9 kbar. Complete metamorphic P-T path was reconstructed using compositional profiling data of a zoned amphibole grain in Sample no. 163. It shows that the actinolite core of the porphyroblast crystallized at relatively lower temperature and pressure conditions of $475^{\circ} \mathrm{C}$ at $2.8 \mathrm{kbar}$ (Fig. 11). Then the amphibole followed a prograde metamorphic evolution up to a maximum temperature of $630^{\circ} \mathrm{C}$ at $5.7 \mathrm{kbar}$. Finally, the amphibole experienced a retrograde re-equilibration under P-T conditions of $515^{\circ} \mathrm{C}$ and $3.2 \mathrm{kbar}$. The predicted P-T path is comparable to that presented by Kohn et al. (2001) for the garnet-grade rocks of the LH in Darondi Valley of central Nepal. However, unlike in the Darondi Valley, present P-T path is the result of at least two unrelated metamorphic events prior and posterior to the India-Asia collision (see discussion).

Four samples (Sample nos. 623, 163, 625, and 180) are suitable for applying hornblende-plagioclase thermometer of Holland and Blundy (1994) as they contain amphiboles with $\mathrm{Na}_{A}>0.02, \mathrm{Al}^{\mathrm{VI}}<1.8$, and $\mathrm{Si}$ in the range of 6.0-7.7, and plagioclases with $X_{\mathrm{An}}<90 \%$. Sample no. 42 contains amphiboles with $\mathrm{Si}>7.7$ and so this sample is not suitable for thermometry. "Programme HbPl 1.2" (Holland and Blundy 1994) was used to calculate average

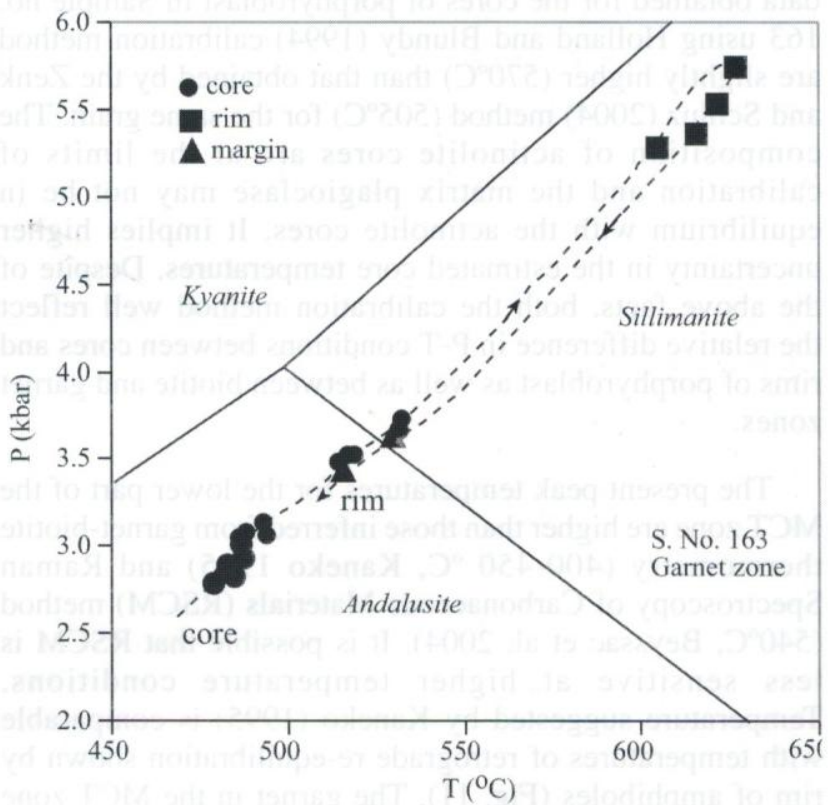

Fig. 11: P-T path estimated from a zoned amphibole grain in sample No. 163. P-T conditions are calculated using revised version (Zenk and Schulz, 2004) of amphibole thermobarometer proposed by Gerya et al. (1977). Arrows indicate P-T evolution trend. Core, rim and margin are defined based on the color of amphibole in thin section (core: light pale green part, rim: dark green part, margin: light pale green part along the grain boundary). The P-T values calculated with this thermobarometer are affected by a minimum error of $\pm 50^{\circ} \mathrm{C} / 1.2 \mathrm{kbar} . \mathrm{Al}_{2} \mathrm{SiO}_{5}$ phase boundaries according to Spear (1993) 
crystallization temperatures of cores and rims porphyroblasts and matrix amphiboles. Pressures were taken as $3.2 \mathrm{kbar}$ for porphyroblast cores and $5.9 \mathrm{kbar}$ for porphyroblast rims and matrix. The average temperatures for cores of porphyroblasts calculated using the Holland and Blundy (1994) calibration method range from 530 to $560^{\circ} \mathrm{C}$ (Table 4). The porphyrobalst rims and matrix were recrystallized at $\sim 575^{\circ} \mathrm{C}$ in the biotite zone and at $\sim 630^{\circ} \mathrm{C}$ in the garnet zone.

The temperature data obtained for the rims of porphyroblasts and matrix amphiboles in Sample no. 163 using Holland and Blundy (1994) and Zenk and Schluz (2004) calibration methods are in the similar range $\left(640^{\circ} \mathrm{C}\right.$ and $620^{\circ} \mathrm{C}$ ). They are also in good agreement with those inferred by the previous workers for the upper part of the MCT zone using garnet-biotite thermometry $\left(\sim 650^{\circ} \mathrm{C}\right.$, Kaneko 1995; $\sim 600^{\circ} \mathrm{C}$, Fraser et al. $2000 ; \sim 650^{\circ} \mathrm{C}$, Catlos et al. $2001 ; \sim 525-625^{\circ} \mathrm{C}$, Kohn et al. 2001). The peak temperatures for the garnet and biotite zones are also similar to those inferred for garnet and biotite zones of the Dalradian sequence (garnet zone $=630^{\circ} \mathrm{C}$; biotite zone $=$ $600^{\circ} \mathrm{C}$, Zenk and Schulz, 2004). However, the temperature data obtained for the cores of porphyroblast in Sample no. 163 using Holland and Blundy (1994) calibration method are slightly higher $\left(570^{\circ} \mathrm{C}\right)$ than that obtained by the Zenk and Schluz (2004) method $\left(505^{\circ} \mathrm{C}\right)$ for the same grain. The composition of actinolite cores are at the limits of calibration and the matrix plagioclase may not be in equilibrium with the actinolite cores. It implies higher uncertainty in the estimated core temperatures. Despite of the above facts, both the calibration method well reflect the relative difference in P-T conditions between cores and rims of porphyroblast as well as between biotite and garnet zones.

The present peak temperatures for the lower part of the MCT zone are higher than those inferred from garnet-biotite thermometry (400-450 ${ }^{\circ} \mathrm{C}$, Kaneko 1995) and Raman Spectroscopy of Carbonaceous Materials (RSCM) method $\left(540^{\circ} \mathrm{C}\right.$, Beyssac et al. 2004). It is possible that RSCM is less sensitive at higher temperature conditions. Temperature suggested by Kaneko (1995) is comparable with temperatures of retrograde re-equilibration shown by rim of amphiboles (Fig. 11). The garnet in the MCT zone show retrograde zoning at the rim (Paudel and Arita 2000), and probably the lower temperatures given by Kaneko (1995) represent the temperatures of retrograde reequilibration rather than the peak temperature. Additionally, validity of garnet-biotite thermometry at temperatures lower than $500^{\circ} \mathrm{C}$ is been questionable (Ferry and Spear 1978; Bhattacharya et al. 1992; Holdaway et al. 1997).

\section{DISCUSSIONS ON TECTONO-METAMORPHIC IMPLICATIONS}

The polymetamorphic evolution of the $\mathrm{LH}$ has been so far inferred based on the indirect evidence such as (i) discordance of illite crystallinity between the Gondwana and pre-Gondwana sediments (Johnson and Oliver 1990; Paudel and Arita 2000), (ii) bimodal compositions of white micas (Paudel and Arita, 2006a) and pre-collisional and post-collisional white mica ages (Oliver et al. 1995). Due to the difficulties in separating detrital white micas from recrystallized ones in clastic metasedimets, the reliability of the above evidences was uncertain. However, the zoned amphiboles in metabasites provide more reliable evidence for the polymetamorphic evolution of the LH.

There are two explanations for the actinolite-hornblende pairs in metabasites: miscibility gap at some pressures and temperatures (see references in Grapes and Graham 1978) or multiple stages of mineral growths (Graham 1974; Holland and Richardson 1979; Liard and Albee 1981). The possibility of miscibility gap can be ruled out for the present case because (i) the amphibole compositions change continuously in the chemical diagrams and do not show any visible compositional gap (Figs. 7a, 7b and 10), and (ii) compositional profiling of zoned amphiboles show continuous change in composition from core to rim (Figs. $7 \mathrm{c}$ and $7 \mathrm{~d}$ ). Therefore, the zoning should result from an incomplete chemical equilibration between cores and rims formed during different metamorphic stages.

The porphyroblasts in lower grade rocks (biotite zone) are either wrapped up or broken apart by the shear planes (Figs. 3b and 3c) clearly showing that they are prekinematic. They are essentially made up of actinolite or magnesio-hornblende. In higher grade rocks (garnet zone), with more prograde recrystallization, the actinolite/ magnesio-hornblende cores are armored by newly crystallized tschermakite/ferro-tschermakite (Figs. 6c, 6d, and 6e). The actinolite/magnesio-hornblende is preserved as relic core of the porphyroblast. The textural and compositional evidence clearly show that the LH experienced a pre-kinematic upper greenschist-facies metamorphism $\left(\mathrm{T} \sim 500-570^{\circ} \mathrm{C}\right.$ and $\left.\mathrm{P}=3 \mathrm{kbar}\right)$ in an intermediate facies-series condition (Figs. 9 and 10).

As the shear foliation $\left(\mathrm{S}_{2}\right)$ in the MCT zone is parallel to the Upper MCT, it is clear that the low-grade metamorphism occurred prior to the thrusting along the Upper MCT. Whether this metamorphism occurred during the Eohimalayan stage or prior to the India-Asia collision can be debated. The ${ }^{40} \mathrm{Ar} /{ }^{39} \mathrm{Ar}$ and $\mathrm{K}-\mathrm{Ar}$ ages of illite from the low-grade metasediments measured by Oliver et al. (1995) clearly show a thermal event prior to the India-Asia collision. Catlos et al. (2000) reported 445 and $252 \mathrm{Ma}$ of $\mathrm{Th}-\mathrm{Pb}$ ages from allanite inclusions in garnet from the footwall of the Upper MCT. Amphibole in metabasites from the LH also show pre-Tertiary cooling ages (Table 5 ). In view of the above ages, we believe that the $\mathrm{LH}$ experienced a thermal event prior to the India-Asia collision in the Tertiary and the pre-kinematic actinolitic amphiboles are the products of pre-Tertiary (pre-Himalayan) thermal event. 
Table 5: Published ${ }^{40} \mathrm{Ar} /{ }^{39} \mathrm{Ar}$ and K-Ar cooling ages of amphiboles from the Lesser Himalaya in Nepal

1. Copeland et al.,(1991), Lesser Himalaya, Buri Gandaki Valley, 40Ar/39Ar hornblende ages

$\begin{array}{lll}\text { Sample No. } & \text { Age }(\mathrm{Ma}) & \text { Dist. From the Upper MCT } \\ \text { AP787 } & 15.2 \pm 0.2 & 100 \mathrm{~m} \\ \text { Ap483 } & 4.1 \pm 1.3 & 500 \mathrm{~m} \\ \text { AP842 } & 94.8 \pm 0.5 & 2 \mathrm{~km} \\ \text { AP343 } & 24.6 \pm 1.9 & 2.5 \mathrm{~km}\end{array}$

2. Krummenacher et al. (1978), Lesser Himalaya, Eastern Nepal, K-Ar hornblende ages

$\begin{array}{lll}\text { KA713 } & 69.4 \pm 2 & \text { KA343 } 253 \pm 15 \\ \text { KA345 } & 96.5 \pm 5 & \text { KA339 } 411 \pm 16 \\ \text { KA341 } & 695 \pm 25 & \end{array}$

3. Bollinger and Jantos (2006), Lesser Himalaya, Far-western Nepal, 40Ar/39Ar hornblende ages

FW0022 $12.5 \pm 0.4$

4. Krummenacher (1966), Lesser Himalaya, Kali Gandaki Valley,
$\mathrm{K}-\mathrm{Ar}$ hornblende ages (Ma)
$819 \pm 80$

It is interesting that the cores of porphyroblasts show an average geothermal gradient of about $55^{\circ} \mathrm{C} / \mathrm{km}$ based on an average temperature of $\sim 540^{\circ} \mathrm{C}$, average pressure of $3 \mathrm{kbar}$, and average crustal density of $2.6 \mathrm{gcm}^{-3}$. This suggests an elevated geothermal gradient in the $\mathrm{LH}$ prior to the Tertiary Himalayan orogeny. The high geothermal gradient prior to the collision can be related to the widespread early Paleozoic (ca. $500 \mathrm{Ma}$ ) felsic magmatism in the Himalaya (Le Fort et al. 1986). However, it remains an open question whether this elevated geotherm resulted from the simple crustal thinning of Gondwana continent (Le Fort and Rai 1999) or an early Paleozoic tectonism (Gehrels et al. 2006).

The inner LH experienced peak metamorphism (Neohimalayan stage) during the Upper MCT activity. Textural records in amphiboles confirm that the matrix amphiboles related to the peak-metamorphic event grew preferentially along the shear planes parallel to the Upper MCT. Although most of porphyroblasts show preferred overgrowth along the foliation, some porphyroblasts show overgrowth across the shear planes. The above evidences show that the Neohimalayan metamorphism was mainly a syn-kinematic event, but the high temperature condition lasted for long time allowing post-kineamtic overgrowth of the amphiboles. Parallelism of shear foliations to the Upper MCT, and presence of stretching and mineral lineation indicate that the second metamorphism is undoubtedly related to the movement along the Upper MCT (Le Fort 1975; Arita 1983; Pêcher 1989). The rocks experienced a peak metamorphic temperature of $\sim 630^{\circ} \mathrm{C}$ at pressure of $\sim 6 \mathrm{kbar}$ during the Neohimalayan stage. This means a geothermal gradient of $\sim 30{ }^{\circ} \mathrm{C} / \mathrm{km}$ if average crustal density is taken as $2.6 \mathrm{gcm}^{-3}$. The greothermal gradient is similar to that shown by b-spacing values of white micas $\left(26^{\circ} \mathrm{C} / \mathrm{km}\right)$ from the MCT zone (Paudel and Arita 2006b). The above geothermal gradient is comparable to that observed in the Dalradian Barrovian metamorphic sequence $\left(22-30^{\circ} \mathrm{C} / \mathrm{km}\right.$, Zenk and Schulz 2004) and in other orogenic domains with Barrovian-type metamorphism (Jamieson et al. 1998).

The systematic chemical changes in syn-kinematic amphiboles, P-T estimations, and petrographic characteristics mentioned above confirm the classical concept of increasing metamorphic grade northwards (structurally upwards) in the LH. The inverted metamorphism is coeval to the shearing due to the Upper MCT activity. Therefore, the metabasites petrology is in favor of the tectono-metamorphic models such as of Le Fort (1975), Arita (1983) and Guillot and Allemand (2002) that relate inverted metamorphism with thrusting along the Upper MCT and coeval inversion of isotherms. 


\section{P. Paudel et al.}

Some studies have shown that diffusivity of structureforming ions as well as the radiogenic isotopes in a single crystal is negligible in amphiboles (Brabander and Giletti 1995; Villa et al. 1996). Therefore, the chemically zoned amphiboles should record radiogenic isotopic characteristics of different metamorphic stages and, hence, the radiometric dating should give a complex age spectra (Villa et al. 2000). Available ${ }^{40} \mathrm{Ar}{ }^{39} \mathrm{Ar}$ and $\mathrm{K}-\mathrm{Ar}$ amphibole cooling ages from the Nepalese LH are given in Table 5. ${ }^{40} \mathrm{Ar}{ }^{39} \mathrm{Ar}$ ages range from $4 \mathrm{Ma}$ to $95 \mathrm{Ma}$ and the $\mathrm{K}$-Ar ages from 69 to $819 \mathrm{Ma}$. The Miocene ages are related to the Neohimalayan metamorphism due to the activity of the Upper MCT. The Pliocene ages may represent the retrograde re-equlibration as well as neocrystallization of amphibole due to the infiltration of hot fluids (Copeland et al. 1991) after the reactivation of the Upper MCT in the Miocene (Harrison et al. 1997). However, the pre-Tertiary ages are suggested to be either due to the extrageneous argon (Copeland et al., 1991) or affected by igneous relict (Krummencher 1978). It is possible that the older cooling ages represent the pre-Tertiary amphiboles. The dispersion of ages from Precambrian to Pliocene indicates that they are the result of mixing of amphiboles of pre-Tertiary and Tertiray amphiboles in different proportions. Therefore, we suggest that the published $\mathrm{K}-\mathrm{Ar}$ and ${ }^{40} \mathrm{Ar} /{ }^{39} \mathrm{Ar}$ amphibole ages from the Nepalese LH which are based on simple, homogeneous mineralogy should be reinterpreted. Combination of microtextural and microchemical data with geochronology could only give reliable cooling ages for the polymetamorphic rocks of the LH.

\section{CONCLUSIONS}

The metabasites of the $\mathrm{LH}$ are tholeiitic basalts emplaced into the clastic sediments and metamorphosed together with the host. The metabasites contain almost constant mineral assemblage of Ca-amphiboles, biotite, plagioclase, quartz, epidote, chlorite and $\mathrm{Fe}-\mathrm{Ti}$ oxides. Modal percentage of amphiboles and biotite increases, and that of epidote and chlorite decreases from south (structurally lower part) to north (structurally higher part) towards the Upper MCT in the LH. Ca-amphiboles in the form of porphyroblast are chemically zoned with actinolite/magnesio-hornblende cores, tschermakite/ferrotschermakite rims and magnesio-hornblende margins, showing their polymetamorphic growth. The actinolite/ magnesio-hornblendes cores are pre-kinematic and were formed most probably prior to the Tertiary Himalayan orogeny. The matrix and rims of porphyroblasts are synkinematic and were formed during shearing along the Upper MCT in the Tertiary. The systematic compositional changes of syn-kinematic amphiboles and P-T estimations together with petrographic characteristics confirm the classical concept of increasing metamorphic grade northwards (structurally upwards) in the LH. The metabasites petrology is in favor of the tectono-metamorphic models that relate inverted metamorphism with thrusting along the Upper MCT and coeval inversion of isotherms. In the context of heterogeneous and polygenetic amphibole mineralogy of metabasites, amphibole cooling ages from the LH based on simple, homogeneous mineralogy should be reinterpreted.

\section{ACKNOWLEDGEMENTS}

This work is a part of post-doctoral research of L.P.P. under fellowship from the Japan Society for the Promotion of Science (JSPS) from 2004-2006. The manuscript was prepared during L. P. P.'s stay at the Tubingen University, Germany as a senior research fellow of the Deutsche Forschungsgemainschaft (German Research Foundation). L. P. P. is grateful to T. Itaya (Okayama University of Science) and E. Appel (Tubingen University) for providing facilities in their laboratories. The authors would thank M. Ikeda (Hokkaido University) for helping in electronprobe microanalyses. The spreadsheet for P-T calculation was kindly provided by B. Schulz. The authors are thankful to Drs. D. Rameshwor Rao and Soibam Ibotomi for their fruitful comments and suggestions for the first draft of manuscript.

\section{REFERENCES}

Ahmad, T., Mukherjee, P. K., and Trivedi, J. R, 1999, Geochemistry of Precambrian mafic magmatic rocks of the western Himalaya, India: Petrogenetic and tectonic implications. Chem. Geol., v. 160, pp.103-119.

Arita, K., 1983, Origin of the inverted metamorphism of the Lower Himalayas, central Nepal. Tectonophysics, v. 95, pp. 43-60.

Arita, K., Hayashi, D., and Yoshida, M., 1982, Geology and structure of the Pokhara-Piuthan area, central Nepal. Jour. Nepal Geol. Soc., Sp. Issue., v. 2, pp. 5-29.

Beyssac, O., Bollinger, L., Avouac, J-P., and Goffé, B., 2004, Thermal metamorphism in the Lesser Himalaya of Nepal determined from Raman spectroscopy of carbonaceous material. Earth Planet Sci. Lett., v. 225, pp. 223-241.

Bhattacharya, A., Mohanty, L., Maji, A., Sen, S. K., and Raith, M., 1992, Non-ideal mixing in the phlogopite-annite binary: constrains from experimental data on $\mathrm{Mg}-\mathrm{Fe}$ partitioning and a reformulation of the biotite-garnet geothrmometer. Contrib. Mineral Petrol., v. 111, pp. 87-93.

Blundy, J. D. and Holland, T. J. B., 1990, Calcic amphibole equilibria and a new amphibole-plagioclase geothermometer. Contrib. Mineral Petrol., v. 104, pp. 208-224.

Bollinger, L., Avouac, J-P., Beyssac, O., Catlos, E. J., Harrison, T. M., Grove, M., Goffé, B., and Sapkota, S., 2004, Thermal structure and exhumation history of the Lesser Himalaya in central Nepal. Tectonics, v. 10.1029/2003TC001564.

Bollinger, L. and Jantos, E., 2006, Evidence for Mio-Pliocene retrograde monazite in the Lesser Himalaya, far western Nepal. Eur. Jour. Mineral, v. 18, pp. 289-297. Not cited in the text

Bouchez, J. L. and Pêcher, A., 1981, The Himalayan Main Central Thrust pile and its quartz rich tectonites in central Nepal. Tectonophysics, v. 78, pp. 23-50.

Brabander, D. J. and Giletti, B. J., 1995, Strontium diffusion kinetics in amphiboles and significance to thermal history determinations. Geochim. Cosmochim. Acta, v. 59, pp. 2223-2238.

Brown, E. H., 1977, The crossite content of Ca-amphibole as a guide to pressure of metamorphism. Jour. Petrol., v. 18, pp. 53-72. 
Brunel, M., 1986, Ductile thrusting in the Himalayas: shear sense criteria and stretching lineations. Tectonics, v. 5, pp. 247-265.

Catlos, E. J., Harrison, T. M., Kohn, M. J., Grove, M., Ryerson, F. J., Manning, C., and Upreti, B. N., 2001, Geochronologic and thermobarometric constraints on the evolution of the Main Central Thrust, central Nepal Himalaya. Jour. Geophys. Res., v. 106, pp. 16177-16204.

Catlos, E. J., Sorensen, S. S., and Harrison, T. M., 2000, Th-Pb ionmicroprobe dating of allanite. Am Mineral, v. 85, pp. 633-648.

Copeland, P., Harrison, T. M., Hodges, K. V., Maruéjol, P., Le Fort, P., and Pêcher, A., 1991, An Early Pliocene disturbance of the Main Central Thrust, central Nepal: implications for Himalayan tectonics. Jour. Geophys Res., v. 96, pp. 8475-8500.

Dhital, M. R, Paudel, L. P, Shrestha, R., Thapa, P. B, Oli, C. B, Paudel, T. R, and Jaisi, D. P, 1998, Geological map (1:50,000 scale) of the area between Kusma, Syangja and Galyang, western Nepal, Lesser Himalaya. Central Dept Geol, Tribhuvan Univ, Kathmandu, Nepal.

Ferry, J. M., and Spear, F. S., 1978, Experimental calibration of the partitioning of $\mathrm{Fe}$ and $\mathrm{Mg}$ between biotite and garnet. Contrib. Mineral Petrol., v. 66, pp. 113-117.

Fraser, G., Worley, B., and Sandiford, M., 2000, High-precision geothermobarometry across the High Himalayan metamorphic sequence, Langtang Valley, Nepal. Jour. Metamorphic Geol., v. 18, pp. 665-681.

Gansser, A., 1964, Geology of the Himalayas. Interscience Publisher, London. 289 p.

Gehrels, G. E., DeCelles, P. G., Ojha, T. P., and Upreti, B. N., 2006, Geologic and $\mathrm{U}-\mathrm{Th}-\mathrm{Pb}$ geochronologic evidence for early Paleozoic tectonism in the Kathmandu thrust sheet, central Nepal Himalaya. GSA Bull., v. 118, pp. 185-198.

Gerya, T. V., Perchuk, L. L., Triboulet, C., Audren, C., and Sez'ko, A. I., 1997, Petrology of the Tumanshet Zonal Metamorphic Complex, Eastern Sayan. Petrology, v. 5, pp. 503-533 (translated from Petrologiya 5:563-595).

Graham, C. M., 1974, Metabasite amphiboles of the Scottish Dalradian. Contrib. Mineral Petrol. v. 47, pp. 165 -185.

Grapes, R. H. and Graham, C. M., 1978, The actinolite-hornblende series in metabasites and so called miscibility gap: a review. Lithos, v. 11, pp. 85-97.

Guillot, S., 1999, An overview of the metamorphic evolution in central Nepal. Jour. Asian Earth Sci., v. 17, pp. 713-725.

Guillot, S. and Allemand, P., 2002, Two-dimensional thermal modeling of the early tectono-metamorphic evolution in central Himalaya. Jour. Geodynamics, v. 34, pp.77-98.

Harrison, T. M., Grove, M., Lovera, O. M., and Catlos, E. J., 1998, A model for the origin of the Himalayan anatexis and inverted metamorphism. Jour. Geophys. Res., v. 103, pp. 27017-27032.

Harrison, T. M., Ryerson, F. J., Le Fort, P., Yin, A., Lovera, O. M., and Catlos, E. J., 1997, A late Miocene-Pliocene origin of the central Himalayan inverted metamorphism. Earth Plan. Sci. Lett., v. 146, pp. E1-E7.

Holdaway, M. J., Mukhopadhyay, B., Dyar, M. D., Guidotti, C. V., and Dutrow, B. L., 1997, Garnet-biotite geothermometry revised: New Margules parameters and a natural specimen data set from Maine. Am. Mineral., v. 82, pp. 582-595.

Holland, T. J. B. and Blundy, J., 1994, Non-ideal interactions in calcic amphiboles and their bearing on amphiboleplagioclase thermometry. Contrib. Mineral. Petrol., v. 116, pp. 443-447.

Holland, T. J. B., Richardson, S. W., 1979, Amphibole zonation in metabasites as a guide to the evolution of metamorphic conditions. Contrib. Mineral. Petrol., v. 70, pp. 143-148.

Hubbard, M. S., 1996, Ductile shear as a cause of inverted metamorphism: example from the Nepal Himalaya. Jour. Geol., v. 104, pp. 493-499.
Hynes, A., 1982, A comparison of amphiboles from medium and low-pressure metabasites. Contrib. Mineral Petrol., v. 81, pp. 119-125.

Imayama, T. and Arita, K., 2007, Nd isotopic data reveal the material and tectonic nature of the Main Central Thrust zone in Nepal Himalaya. Tectonophysics, doi: 10.1016/j.tecto.2007.11.051

Jamieson, R. A, Beaumont, C., Fullsack P, and Lee, B., 1998 Barrovian regional metamorphism: where's the heat? In: Treloar PJ, O'Brien PJ (eds) What drives metamorphism and metamorphic reactions? Geol Soc London Sp Publ 138:23-51.

Johnson, MRW, Oliver GJH (1990) Precollisional and post collisional thermal events in the Himalaya. Geology 18:753-756

Kaneko, Y., 1995, Thermal structure in the Annapurna region, central Nepal Himalaya: implication for the inverted metamorphism. Jour. Mineral Petrol Economic Geol 90:143-154.

Kohn, M. J., Catlos, E. J., Ryerson, F. J., and Harrison, T. M., 2001, Pressure-temperture-time path discontinuity in the Main Central thrust zone, central Nepal. Geology, v. 29, pp. 571-574.

Krummenacher, D., 1966, Népal Central: géochronométrie des series de l'Himalaya. Bull. Schweiz Mineral Petrogr. Mitteil., v. 46, pp. 43-54. (Not cited in the text??

Krummenacher, D., Basett, A. M., Kingery, F. A., and Layne, H. F., 1978, Petrology, metamorphism and K/Ar age determinations in Eastern Nepal. In: Saklani PS (ed.) Tectonic Geology of the Himalaya, Today and Tomorrow's Printers and Publishers, New Delhi. (Not cited in the text??

Le Fort, P., 1975, Himalayas: the collided range, present knowledge of the continental arc. Am. Jour. Sci., v. 275A, pp. 1-44.

Le Fort, P., Debon, F., Pêcher, A., Sonet, J., and Vidal, P., 1986, The 500 Ma magmatic event in Alpine southern Asia, a thermal episode at Gondwana scale. Sciences de la Terre, v. 47, pp. 191-209.

Le Fort, P. and Rai, S. M., 1999, Pre-Tertiary felsic magmatism of the Nepal Himalaya : recycling of the continental crust. Jour. Asian Earth Sci., v. 17, pp. 607-628.

Leake, B. E., Woolley, A. R., Arps, C. E. S., Birch, W. D., Gilbert, M. C., Grice, J. D., Hawthorne, F. C., Kato, F. C., Kisch, H. J., Krichovichev, V. G., Linthout, K., Laird, J., Mandarino, J. A, Maresch, W. V., Nickel, E. H., Rock, N. M. S., Schumacher, J. C., Smith, D. C., Stephenson, N. C. N., Ungaretti, L., Whittake, E. J. W., and Youzhi, G., 1997, Nomenclature of amphiboles: report of the subcommittee on amphiboles of the International Mineralogical Association, commission on new minerals and mineral names. Am. Mineral, v. 82, pp. 1019-1037.

Liard, J., 1980, Phase equilibria in mafic schist from Vermont. Jour. Petrology, v. 21, pp. 1-37.

Liard, J. and Albee, A. L., 1981, Pressure, temperature, and time indicators in mafic schist: their application to reconstructing the polymetamorphic history of Vermont. Am. Jour. Sci., v. 281, pp. $127-175$.

Macfarlane, A. M., 1995, An evaluation of the inverted metamorphic gradient at Langtang National Park, central Nepal Himalaya. Jour. Metamorphic Geol., v. 13, pp. 595-612.

Miyashiro, A., 1973, Metamorphism and Metamorphic Belts. Allen and Unwin, London

Oliver, G. H. J., Johnson, M. R. W., and Fallick, A. E., 1995, Age of metamorphism in the Lesser Himalaya and the Main Central Thrust zone, Garhwal India: results of illite crystallinity, ${ }^{40} \mathrm{Ar} /$ ${ }^{39}$ Ar fusion and K-Ar studies. Geol. Mag., v. 132, pp. 139-149.

Paudel, L. P. and Arita, K., 2000, Tectonic and polymetamorphic history of the Lesser Himalaya in central Nepal. Jour. Asian Earth Sci., v. 18, pp. 561-584.

Paudel, L. P. and Arita, K., 2002, Locating the Main Central Thrust in central Nepal using lithologic, microstructural and metamorphic criteria. Jour. Nepal Geol. Soc., v. 26, pp. 29-42. 
Paudel, L. P. and Arita, K., 2006a, Thermal evolution of the Lesser Himalaya, central Nepal: Insights from K-white micas compositional variation. Gondwana Res., v. 9, pp. 409-425.

Paudel, L. P. and Arita, K., 2006b, The b-spacing values of white micas and their metamorphic implications in the Lesser Himalaya of central Nepal. Jour. Asian Earth Sci., v. 27, pp. 10-24.

Paudel, L. P., Itaya, T., and Arita, K., 2005, Electron micro-probe analyses of amphiboles in metabasic rocks of the central Nepal Lesser Himalaya. Bull Research Institute Natural Sci., Okayama Univ. Sci., v. 31, pp. 17-25.

Pêcher, A., 1977, Geology of the Nepal Himalaya: deformation and petrography in the Main Central Thrust zone. Ecologie géologie l'Himalaya, CNRS Paris, v. 268, pp. 301-318.

Pêcher, A., 1989, The metamorphism in central Himalaya. Jour. Metamorphic Geol., v. 7, pp. 31-41.

Pêcher, A. and Le Fort, P., 1986, The metamorphism in Central Himalaya, its relations with the thrust tectonics. Sciences de la Terre, v. 47, pp. 285-309.

Pognante, U. and Benna, P., 1993, Metamorphic zonation, migmatization, and leucogranites along the Everest transect of Eastern Nepal and Tibet: record of an exhumation history. In: Treloar PJ, Searle MP (eds.), Himalayan Tectonics, Geol. Soc. London, Spec. Publication, v. 74, pp. 328-340.

Raase, P., 1974, $\mathrm{Al}$ and $\mathrm{Ti}$ contents of hornblende, indicators of pressure and temperature of regional metamorphism. Contrib. Mineral Petrol., v. 45, pp. 231-236.

Rai, S. M., Guillot, S., Le Fort, P., and Upreti, B. N., 1998, Pressuretemperature evolution in the Kathmandu and Gosainkund regions, central Nepal. Jour. Asian Earth Sci., v. 16, pp. 283-298.

Sakai, H., 1985, Geology of the Kali Gandaki supergroup of the Lesser Himalaya in Nepal. Memoirs of the Faculty of Science, Kyushu University (Japan). Series D, Geology, v. XXV, pp. 337-397.

Schumacher, J. C., 1997, The estimation of the proportion of ferric iron in the electron microprobe analysis of amphiboles. The Canadian Mineral, v. 35, pp. 238-246.
Searle, M. P. and Rex, D. C., 1989, Thermal model for the Zanskar Himalaya. Jour. Metamorphic Geol., v. 7, pp. 127-134.

Spear, F. S., 1980, NaSi-CaAl exchange equilibrium between plagioclase and amphibole: an empirical model. Contrib. Mineral Petrol., v. 80, pp. 140-146.

Spear, F. S., 1993, Metamorphic Phase Equilibria and PressureTemperature-Time Paths. Monograph Series 1, Mineralogical Society of America, Washington, D.C. (Not cited in the text

Stöcklin, J., 1980, Geology of Nepal and its regional frame. Jour. Geol. Soc. London, v. 137, pp. 1-34.

Tanaka, R. and Orihashi, Y., 1997, XRF Analysis of major and trace elements for silicate rocks using low dilution ratio fused glass. Hokkaido University Earth and Planetary Science Technical Report, v. 2, pp. 1-20.

Triboulet, C., Thie blemont, D., and Audren, C., 1992, The (Na$\mathrm{Ca}$ a mphibole-albite-chlorite-epidote-quartz geothermobarometer in the system S-A-FM-C-N-H O. 2. Applications to metabasic rocks in different metamorphic settings. Jour. Metamorphic Geol., v. 10, pp: 557-566.

Upreti, B. N., 1999, An overview of the stratigraphy and tectonics of the Nepal Himalaya. Jour. Asian Earth Sci., v. 17, pp. 577 606. (not cited in the text

Villa, I. M., Grobéty, B., Kelly, S. P., Trigila, R., and Wieler, R., 1996, Assessing Ar transport paths and mechanisms in the McClure Mountains hornblende. Contrib. Mineral Petrol., v. 126, pp. $67-80$.

Villa, I. M., Hermann, J., Müntener, Z., and Trommsdorff, V., 2000, ${ }^{40} \mathrm{Ar}-{ }^{39} \mathrm{Ar}$ dating of multiply zoned amphibole generations (Malenco, Italian Alps). Contrib. Mineral Petrol., v. 140, pp. 363-381.

Winchester, J. A. and Floyd, P. A., 1977, Geochemical discrimination of different magma series and their differentiation products using immobile elements. Chem. Geol., v. 20, pp. 325-344.

Zenk, M. and Schulz, B., 2004, Zoned Ca-amphiboles and related $\mathrm{P}-\mathrm{T}$ evolution in metabasites from the classical Barrovian metamorphic zones in Scotland. Mineral Mag., v. 68, pp. 769-786. 